\title{
Fracture in distortion gradient plasticity
}

\author{
Sandra Fuentes-Alonso ${ }^{a}$, Emilio Martínez-Pañedab,c,* \\ ${ }^{a}$ Department of Construction and Manufacturing Engineering, University of Oviedo, \\ Gijón 33203, Spain \\ ${ }^{b}$ Department of Civil and Environmental Engineering, Imperial College London, London \\ $S W 72 A Z, U K$ \\ ${ }^{c}$ Department of Engineering, Cambridge University, CB2 1PZ Cambridge, UK
}

\begin{abstract}
Due to its superior modelling capabilities, there is an increasing interest in distortion gradient plasticity theory, where the role of the plastic spin is accounted for in the free energy and the dissipation. In this work, distortion gradient plasticity is used to gain insight into material deformation ahead of a crack tip. This also constitutes the first fracture mechanics analysis of gradient plasticity theories adopting Nye's tensor as primal kinematic variable. First, the asymptotic nature of crack tip fields is analytically investigated. A generalised $J$-integral is defined and employed to determine the power of the singularity. We show that an inner elastic region exists, adjacent to the crack tip, where elastic strains dominate plastic strains and Cauchy stresses follow the linear elastic $r^{-1 / 2}$ stress singularity. This finding is verified by detailed finite element analyses using a new numerical framework, which builds upon a viscoplastic constitutive law that enables capturing both rate-dependent and rate-independent behaviour in a computationally efficient manner. Numeri-
\end{abstract}

\footnotetext{
${ }^{*}$ Corresponding author.

Email address: e.martinez-paneda@imperial.ac.uk (Emilio Martínez-Pañeda)
} 
cal analysis is used to gain further insight into the stress elevation predicted by distortion gradient plasticity, relative to conventional $J_{2}$ plasticity, and the influence of the plastic spin under both mode I and mixed-mode fracture conditions. It is found that Nye's tensor contributions have a weaker effect in elevating the stresses in the plastic region, while predicting the same asymptotic behaviour as constitutive choices based on the plastic strain gradient tensor. A minor sensitivity to $\chi$, the parameter governing the dissipation due to the plastic spin, is observed. Finally, distortion gradient plasticity and suitable higher order boundary conditions are used to appropriately model the phenomenon of brittle failure along elastic-plastic material interfaces. We reproduce paradigmatic experiments on niobium-sapphire interfaces and show that the combination of strain gradient hardening and dislocation blockage leads to interface crack tip stresses that are larger than the theoretical lattice strength, rationalising cleavage in the presence of plasticity at bi-material interfaces.

Keywords:

Distortion gradient plasticity, finite element method, crack tip mechanics, size effects, higher order theories

\section{Introduction}

In recent years, there has been an increasing interest in characterising the behaviour of metals at the micrometer scale. Examples are found in microelectromechanical systems (MEMS), microelectronic components, and thin film applications. A wide array of micron scale experiments have revealed that metals display pronounced size effects when deformed non-uniformly 
into the plastic range (see Ref. [1] for a review). Notable pioneering examples are the wire torsion experiments by Fleck and co-workers [2], the nanoindentation measurements by Nix and Gao [3], and the bending of foils by Stölken and Evans [4]. Fleck et al. [2] tested very thin copper wires (with radius varying from 6 to $85 \mu \mathrm{m}$ ) under both uniaxial tension and torsion. Results revealed only a minor influence of specimen size on tensile behaviour but a systematic increase in torsional strengthening with decreasing wire diameter. Nix and Gao [3] found a linear relation between the indentation depth and the hardness of single crystal and cold worked polycrystalline copper. This size effect becomes negligible as the indentation depth is increased beyond a characteristic length on the order of micrometers. Stölken and Evans [4] conducted micro-bending tests on nickel foils of different thicknesses, showing that thinner specimens are stronger and strain harden more than thicker ones. Uniaxial tension tests were also conducted and, as in the work by Fleck et al. [2], almost no influence of specimen size is observed. Thus, the smaller is harder or smaller is stronger trends observed in the aforementioned micron scale experiments are intrinsically associated with the presence of strain gradients. In terms of the underpinning dislocation phenomena, work hardening is controlled by the total density of dislocations, part of which is related to the gradients of plastic strain. Thereby, dislocation storage governing material hardening is due to: (i) dislocations that trap each other in a random way and (ii) dislocations required for compatible deformation of various parts of the crystal [5]. The latter are referred to as Geometrically Necessary Dislocations (GNDs) while the former are named Statistically Stored Dislocations (SSDs). GNDs do not contribute to plastic strain but to material work hard- 
ening by acting as obstacles to the motion of SSDs. This extra storage of dislocations associated with gradients of plastic strain will manifest its influence when the characteristic length of deformation becomes sufficiently small.

Experimental evidence of strain gradient hardening has been accompanied by a vast literature on the development of enriched isotropic plasticity models, so-called strain gradient plasticity theories (see, e.g. [6 13] and references therein). Consistent with experimental observations, theoretical models are cast in a form where the plastic work depends on both strains and strain gradients; introducing a material length scale $\ell$, and reducing to conventional plasticity when the length scales of the imposed deformation gradients are large compared to $\ell$. Recent theoretical developments have been aimed at capturing a wide range of experimental observations. Thus, modern strain gradient plasticity formulations consider both dissipative (or unrecoverable) and energetic (or recoverable) gradient contributions, to capture both the strengthening and hardening effects observed [11, 12, 14]. In addition, the form of the free energy has received particular interest [15 20]. Gradient effects are accounted for by means of an additional contribution to the Helmholtz free energy, the so-called defect energy. Choices include the use of plastic strains or Nye's tensor as primal higher order kinematic variables, considering one or more invariants of the primal variable, and exploring less-than-quadratic forms of the defect energy. Also, increased attention has been focused on the need to account for the plastic spin in recent years, as originally proposed by Gurtin [21], to properly describe plastic flow incompatibility and the associated dislocation densities. This class of gradient 
plasticity models is referred to as distortion gradient plasticity, as it builds upon the incompatibility of the plastic part of the displacement gradient and the macroscopic characterisation of the Burgers vector [21 24] to rigorously define Nye's tensor as:

$$
\alpha_{i j}=\epsilon_{j k l} \gamma_{i l, k}^{p} \quad\left(\boldsymbol{\alpha}=\operatorname{curl} \gamma^{p}\right)
$$

where $\gamma_{i j}^{p}$ is the plastic distortion - the plastic part of the displacement gradient. The increasing popularity of distortion gradient plasticity lies on its superior modelling capabilities. As shown by Bardella and co-workers [2527] and Poh and Peerlings [28], the contribution of the non-symmetric plastic part of the displacement gradient plays a fundamental role in capturing essential features of crystal plasticity. Moreover, Poh and Peerlings [28] showed that the localization phenomenon that takes place in Bittencourt et al. [29] composite unit cell benchmark problem can only be reproduced by distortion gradient plasticity. Other recent works involve the development of new homogenization formulations [30, 31] and finite element schemes [32 34]. However, the implications of distortion gradient plasticity on crack tip mechanics remain to be addressed.

Strain gradient effects are typically characterised via micro-scale experiments but are present in any boundary value problem where the strain varies over microns. This is the case of fracture mechanics problems where, independently of the size of the cracked sample, the plastic zone adjacent to the crack tip is typically small and contains large spatial gradients of plastic deformation. The analysis of stationary cracks with strain gradient plasticity reveals that local strain gradient hardening elevates crack tip stresses far 
beyond conventional plasticity predictions [35 38]. The stress elevation predicted by strain gradient plasticity provides a rationale for brittle fracture in the presence of plasticity [35, 39] and has important implications for a number of structural integrity problems, such as hydrogen embrittlement [40, 41], fatigue damage [42, 43], and low-temperature cleavage [44, 45]. In some of these applications, quantitative differences are of utmost importance. For example in hydrogen embrittlement, where the crack tip hydrogen content has an exponential dependence on the hydrostatic stress [46]. However, fracture studies have focused on a few gradient plasticity models and the impact of recent theoretical developments is yet to be investigated.

In this work, distortion gradient plasticity is used for the first time to model crack tip behaviour. Also, the role of Nye's tensor on fracture mechanics is first elucidated. We combine analytical insight into the asymptotic stress field with detailed finite element analysis of crack tip fields under mode I and mixed mode fracture conditions, revealing some remarkable results. The remaining of the manuscript starts by introducing the flow theory of distortion gradient plasticity (Section 2). This is followed by an analytical investigation of the nature of the asymptotic crack tip solution in Section 3 . Our analysis reveals the existence of an elastic region close to the crack tip, reminiscent of a dislocation-free zone. This feature is confirmed by numerical computations and extends the recent findings by Martínez-Pañeda and Fleck [47] to distortion gradient plasticity and strain gradient theories based on Nye's dislocation density tensor. In Section 4, the finite element framework is described, including the development of a new viscoplastic potential. The 
numerical model is then used in Section 5 to characterize the influence of the plastic spin and material parameters on crack tip fields. It is shown that the use of a defect energy based on Nye's tensor leads to a much weaker stress elevation relative to defect energies employing the plastic strain tensor as primal variable. Finally, insight is shed into the conundrum of atomic decohesion at metal-ceramic interfaces by modelling the classic experiments by Elssner et al. [48] and Korn et al. [49]. The manuscript ends with concluding remarks in Section 6 .

\section{The flow theory of distortion plasticity plasticity}

The equations of this section refer to the mechanical response of a body occupying a space region $\Omega$ with an external surface $S$ of outward normal $n_{i}$. More details about the higher order theory of distortion gradient plasticity can be found in Ref. [21].

\subsection{Variational principles and balance equations}

Within a small strain formulation, the displacement gradient $u_{i, j}$ can be decomposed into its elastic and plastic parts:

$$
u_{i, j}=\gamma_{i j}^{e}+\gamma_{i j}^{p}
$$

Where $\gamma_{i j}^{p}$, the plastic distortion, which characterises the evolution of dislocations and other defects through the crystal structure, may in turn be decomposed into its symmetric and skew parts:

$$
\gamma_{i j}^{p}=\varepsilon_{i j}^{p}+\vartheta_{i j}^{p}
$$


Unlike the plastic strain field $\varepsilon_{i j}^{p}$, the plastic rotation $\vartheta_{i j}^{p}$ is essentially irrelevant in a conventional theory. However, as pointed out by Gurtin [21], phenomenological models involving Nye's dislocation density tensor $\alpha_{i j}$ as primal higher order kinematic variable,

$$
\alpha_{i j}=\epsilon_{j k l} \gamma_{i l, k}^{p} \quad\left(\boldsymbol{\alpha}=\operatorname{curl} \boldsymbol{\gamma}^{p}\right)
$$

must account for the plastic spin since the macroscopic characterisation of the Burgers vector involves both the symmetric and skew parts of the plastic distortion

$$
\epsilon_{j k l} \gamma_{i l, k}^{p}=\epsilon_{j k l} \varepsilon_{i l, k}^{p}+\epsilon_{j k l} \vartheta_{i l, k}^{p} \quad\left(\operatorname{curl} \boldsymbol{\gamma}^{p}=\operatorname{curl} \boldsymbol{\varepsilon}^{p}+\operatorname{curl} \boldsymbol{\vartheta}^{p}\right)
$$

with $\epsilon_{j k l}$ denoting the alternating symbol. The internal virtual work reads:

$$
\delta W_{i}=\int_{\Omega}\left(\sigma_{i j} \delta \varepsilon_{i j}^{e}+\zeta_{i j} \delta \alpha_{i j}+S_{i j} \delta \gamma_{i j}^{p}+\tau_{i j k} \delta \varepsilon_{i j, k}^{p}\right) \mathrm{d} V
$$

where the Cauchy stress is denoted by $\sigma_{i j}$. In addition to conventional stresses, the principle of virtual work incorporates the so-called micro-stress tensor, $S_{i j}$ (work conjugate to the plastic distortion, $\gamma_{i j}^{p}$ ), the defect stress $\zeta_{i j}$ (work conjugate to Nye's tensor $\alpha_{i j}$, the curl of the plastic distortion) and the - here, purely dissipative - higher order stress tensor, $\tau_{i j k}$ (work conjugate to the plastic strain gradients $\varepsilon_{i j, k}^{p}$ ). By taking into account that the micro-stress tensor can be decomposed into its symmetric and skew parts: $S_{i j}=q_{i j}+\omega_{i j}$, the internal virtual work statement can be expressed as:

$$
\delta W_{i}=\int_{\Omega}\left(\sigma_{i j} \delta \varepsilon_{i j}+\zeta_{i j} \delta \alpha_{i j}+\left(q_{i j}-\sigma_{i j}^{\prime}\right) \delta \varepsilon_{i j}^{p}+\omega_{i j} \delta \vartheta_{i j}^{p}+\tau_{i j k} \delta \varepsilon_{i j, k}^{p}\right) \mathrm{d} V
$$

with the prime symbol ' denoting deviatoric quantities. Applying Gauss' 
divergence theorem to (7) renders:

$$
\begin{aligned}
\delta W_{i} & =\int_{S}\left(\sigma_{i j} n_{j} \delta u_{i}+\left(\Upsilon_{i j}^{\prime}+\tau_{i j k} n_{k}\right) \delta \varepsilon_{i j}^{p}+\Delta_{i j} \delta \vartheta_{i j}^{p}\right) \mathrm{d} S \\
& -\int_{\Omega}\left(\sigma_{i j, j} \delta u_{i}-\left(q_{i j}-\sigma_{i j}^{\prime}-\tau_{i j k, k}+\eta_{i j}^{\prime}\right) \delta \varepsilon_{i j}^{p}-\left(\omega_{i j}+\varphi_{i j}\right) \delta \vartheta_{i j}^{p}\right) \mathrm{d} V
\end{aligned}
$$

where $\eta_{i j}$ and $\varphi_{i j}$ are, respectively, the symmetric and skew-symmetric parts of the curl of the defect stress $\xi_{i j}=\epsilon_{j k l} \zeta_{i l, k}=\eta_{i j}+\varphi_{i j}$; and equivalently, $\Upsilon_{i j}$ and $\Delta_{i j}$ respectively denote the symmetric and skew-symmetric parts of the cross product of the defect stress and the outward normal $\Gamma_{i j}=\epsilon_{j k l} \zeta_{i l} n_{k}=\Upsilon_{i j}+\Delta_{i j}$. Since the volume integral in (8) should vanish for arbitrary variations, three sets of equilibrium equations are readily obtained:

$$
\begin{aligned}
& \sigma_{i j, j}=0 \\
& q_{i j}-\sigma_{i j}^{\prime}-\tau_{i j k, k}+\eta_{i j}^{\prime}=0 \\
& \omega_{i j}+\varphi_{i j}=0
\end{aligned}
$$

Now, identifying the surface in (8) as part of the external work and considering (7), the Principle Virtual Work reads:

$$
\begin{aligned}
& \int_{\Omega}\left(\sigma_{i j} \delta \varepsilon_{i j}+\zeta_{i j} \delta \alpha_{i j}+\left(q_{i j}-\sigma_{i j}^{\prime}\right) \delta \varepsilon_{i j}^{p}+\omega_{i j} \delta \vartheta_{i j}^{p}+\tau_{i j k} \delta \varepsilon_{i j, k}^{p}\right) \mathrm{d} V \\
& =\int_{S}\left(T_{i} \delta u_{i}+t_{i j}^{\varepsilon} \delta \varepsilon_{i j}^{p}+t_{i j}^{\vartheta} \delta \vartheta_{i j}^{p}\right) \mathrm{d} S
\end{aligned}
$$

where $T_{i}$ are the conventional tractions, work conjugate to the displacements, while $t_{i j}^{\varepsilon}$ and $t_{i j}^{\vartheta}$ denote the higher order tractions work conjugate to plastic strains $\varepsilon_{i j}^{p}$ and plastic rotations $\vartheta_{i j}^{p}$, respectively. Accordingly, considering 
(8), the natural boundary conditions read:

$$
\begin{aligned}
& T_{i}=\sigma_{i j} n_{j} \\
& \Upsilon_{i j}^{\prime}+\tau_{i j k} n_{k}=t_{i j}^{\varepsilon} \\
& \Delta_{i j}=t_{i j}^{\vartheta}
\end{aligned}
$$

\subsection{Energetic contributions}

In order to account for the influence of GNDs, the free energy is chosen to depend on both the elastic strain $\varepsilon_{i j}^{e}$ and Nye's tensor $\alpha_{i j}$ :

$$
\Psi=\frac{1}{2} C_{i j k l} \varepsilon_{i j}^{e} \varepsilon_{k l}^{e}+\Phi\left(\alpha_{i j}\right)
$$

with $C_{i j k l}$ being the elastic stiffness and $\Phi\left(\alpha_{i j}\right)$ the defect energy that accounts for the recoverable mechanisms associated with the development of GNDs. The widely used quadratic form of the defect energy is adopted

$$
\Phi\left(\alpha_{i j}\right)=\frac{1}{2} \mu L_{E}^{2} \alpha_{i j} \alpha_{i j}
$$

but one should note that exploring other options may lead to further modelling capabilities [15 20]. Accordingly, the defect stress equals:

$$
\zeta_{i j}=\frac{\partial \Phi\left(\alpha_{i j}\right)}{\partial \alpha_{i j}}=\mu L_{E}^{2} \alpha_{i j}
$$

with $\mu$ being the shear modulus and $L_{E}$ the energetic material length scale.

\subsection{Dissipative contributions}

A gradient-enhanced phenomenological effective plastic flow rate is defined,

$$
\dot{E}^{p}=\sqrt{\frac{2}{3} \dot{\varepsilon}_{i j}^{p} \dot{\varepsilon}_{i j}^{p}+\chi \dot{\vartheta}_{i j}^{p} \dot{j}_{i j}^{p}+\frac{2}{3} L_{D}^{2} \dot{\varepsilon}_{i j, k}^{p} \dot{\varepsilon}_{i j, k}^{p}}
$$


where $L_{D}$ is a dissipative length parameter and $\chi$ is the parameter governing the dissipation due to the plastic spin. Bardella [26] has analytically identified the value of $\chi$ that captures the mechanical response of a crystal subjected to multi-slip under simple shear:

$$
\chi=\left[\frac{3}{2}+\frac{\sigma_{Y}}{\mu \varepsilon_{Y}}\left(\frac{L_{D}}{L_{E}}\right)^{2}\right]^{-1}
$$

being $\sigma_{0}$ and $\varepsilon_{0}$ non-negative material parameters, which implies a value for $\chi$ bounded between 0 and $2 / 3$. The flow resistance $\Sigma$, work conjugate to $\dot{E}^{p}$, is given by

$$
\Sigma=\sqrt{\frac{3}{2} q_{i j} q_{i j}+\frac{1}{\chi} \omega_{i j} \omega_{i j}+\frac{3}{2 L_{D}^{2}} \tau_{i j k} \tau_{i j k}}
$$

Such that the unrecoverable stresses equal

$$
q_{i j}=\frac{2}{3} \frac{\Sigma}{\dot{E}^{p}} \dot{\varepsilon}_{i j}^{p}, \quad \omega_{i j}=\chi \frac{\Sigma}{\dot{E}^{p}} \dot{\vartheta}_{i j}^{p}, \quad \tau_{i j k}=\frac{2}{3} L_{D}^{2} \frac{\Sigma}{\dot{E}^{p}} \dot{\varepsilon}_{i j, k}^{p}
$$

And consequently the second law of thermodynamics is fulfilled by relating finite stress measures with rates of plastic deformation, in what is referred to as a non-incremental form:

$$
q_{i j} \dot{\varepsilon}_{i j}^{p}+\omega_{i j} \dot{\vartheta}_{i j}^{p}+\tau_{i j k} \dot{\varepsilon}_{i j, k} \equiv \Sigma \dot{E}^{p}>0
$$

\section{Asymptotic analysis of crack tip fields}

We begin our study by conducting an asymptotic analysis of the relevant fields at the crack tip under mode I fracture conditions. Consider a crack in a $2 \mathrm{D}$ space, with its tip at the origin of a polar coordinate system $(r, \theta)$. We will assume that the plastic distortion field $\gamma_{i j}^{p}$ is continuous and differentiable, with an asymptotic solution that behaves as follows:

$$
\gamma_{i j}^{p} \sim r^{\beta} f_{i j}(\theta)
$$


for $r \rightarrow 0$. By deriving a generalized $J$-integral for distortion gradient plasticity, the order of the singularity (index $\beta$ ) will be determined using energy boundness arguments and its implications for the behaviour of crack tip stresses investigated.

\subsection{Deformation theory solid}

In a deformation theory context, in the absence of conventional and higher order tractions, the total potential energy assumes the form,

$$
U\left(u_{i}, \varepsilon_{i j}^{p}, \vartheta_{i j}^{p}, \varepsilon_{i j, k}^{p}, \alpha_{i j}\right)=\int_{V}\left[\Psi\left(u_{i}, \alpha_{i j}\right)+\varphi\left(\varepsilon_{i j}^{p}, \vartheta_{i j}^{p}, \varepsilon_{i j, k}^{p}\right)\right] \mathrm{d} V
$$

with the free energy being given by,

$$
\Psi\left(u_{i}, \alpha_{i j}\right)=\Psi^{e}\left(u_{i}\right)+\Phi\left(\alpha_{i j}\right)=\frac{1}{2}\left(\varepsilon_{i j}-\varepsilon_{i j}^{p}\right) C_{i j k l}\left(\varepsilon_{k l}-\varepsilon_{k l}^{p}\right)+\frac{1}{2} \mu L_{E}^{2} \alpha_{i j} \alpha_{i j}
$$

Here, $\Psi^{e}$ denotes the elastic free energy. Thereby, the Cauchy stresses are derived as,

$$
\sigma_{i j}=\frac{\partial \Psi}{\partial \varepsilon_{i j}^{e}}=C_{i j k l}\left(\varepsilon_{k l}-\varepsilon_{k l}^{p}\right)
$$

and the so-called defect stress $\zeta_{i j}$ is given by (18).

On the other hand, the dissipation potential $\varphi$ is given by,

$$
\varphi\left(\varepsilon_{i j}^{p}, \vartheta_{i j}^{p}, \varepsilon_{i j, k}^{p}\right)=\frac{\sigma_{Y} \varepsilon_{Y}}{N+1}\left(\frac{E^{p}\left(\varepsilon_{i j}^{p}, \vartheta_{i j}^{p}, \varepsilon_{i j, k}^{p}\right)}{\varepsilon_{Y}}\right)^{N+1}
$$

where $\sigma_{Y}$ is the yield stress, $\varepsilon_{Y}$ is the yield strain, and

$$
\left(E^{p}\right)^{2}=\frac{2}{3} \varepsilon_{i j}^{p} \varepsilon_{i j}^{p}+\chi \vartheta_{i j}^{p} \vartheta_{i j}^{p}+\frac{2}{3} L_{D}^{2} \varepsilon_{i j, k}^{p} \varepsilon_{i j, k}^{p}
$$

The choice (28) implies that a homogeneous hardening law relates $E^{p}$ with its work conjugate, the effective stress $\Sigma$,

$$
\Sigma=\sigma_{Y}\left(\frac{E^{p}}{\varepsilon_{Y}}\right)^{N}=\Sigma_{0}\left(E^{p}\right)^{N}
$$


where $0 \leq N \leq 1$ is the strain hardening exponent. Hence, the dissipation potential reads,

$$
\varphi\left(\varepsilon_{i j}^{p}, \vartheta_{i j}^{p}, \varepsilon_{i j, k}^{p}\right)=\frac{\Sigma E^{p}}{N+1}
$$

Accordingly, the constitutive relations for the deformation theory solid can be readily derived as

$$
\begin{gathered}
q_{i j}=\frac{\partial \varphi}{\partial \varepsilon_{i j}^{p}}=\sigma_{Y}\left(\frac{E^{p}}{\varepsilon_{Y}}\right)^{N} \frac{2}{3} \frac{\varepsilon_{i j}^{p}}{E^{p}}=\frac{2}{3} \frac{\Sigma}{E^{p}} \varepsilon_{i j}^{p} \\
\omega_{i j}=\frac{\partial \varphi}{\partial \vartheta_{i j}^{p}}=\sigma_{Y}\left(\frac{E^{p}}{\varepsilon_{Y}}\right)^{N} \chi \frac{\vartheta_{i j}^{p}}{E^{p}}=\chi \frac{\Sigma}{E^{p}} \vartheta_{i j}^{p} \\
\tau_{i j k}=\frac{\partial \varphi}{\partial \varepsilon_{i j, k}^{p}}=\sigma_{Y}\left(\frac{E^{p}}{\varepsilon_{Y}}\right)^{N} L_{D}^{2} \frac{2}{3} \frac{\varepsilon_{i j, k}^{p}}{E^{p}}=\frac{2}{3} L_{D}^{2} \frac{\Sigma}{E^{p}} \varepsilon_{i j, k}^{p}
\end{gathered}
$$

\subsection{A generalized J-integral for distortion gradient plasticity}

We proceed to define a generalized $J$-integral for distortion gradient plasticity. Consider a Cartesian coordinate system $(x, y)$ with the crack tip at the origin and the crack plane along the negative $x$ axis. Defining $J$ as the energy release rate per unit crack extension and $w$ as the strain energy density of the solid, an evaluation of $J$ over a contour $\Gamma$ that encloses the crack tip gives

$$
J=\int_{\Gamma}\left(w n_{x}-\sigma_{i j} n_{j} u_{i, x}-t_{i j}^{\vartheta} \vartheta_{i j}^{p}-t_{i j}^{\varepsilon} \varepsilon_{i j}^{p}\right) \mathrm{d} S
$$

The derivation and proof are straightforward and follow the works by Eshelby [50] and Rice [51] in the context of conventional deformation solids, and the recent work by Martínez-Pañeda and Fleck [47] for strain gradient solids. Note that the existence of a $J$-integral implies that total strain energy density of the solid will asymptotically behave as $w \sim J / r$ so as to give a finite 
energy release rate $J$ at the crack tip. Following the notation of Section 3.1 , this energy boundness constraint can be expressed as:

$$
\Psi^{e}\left(\varepsilon_{i j}^{e}\right)+\Phi\left(\alpha_{i j}\right)+\varphi\left(\varepsilon_{i j}^{p}, \vartheta_{i j}^{p}, \varepsilon_{i j, k}^{p}\right) \sim \frac{J}{r}
$$

for $r \rightarrow 0$.

\subsection{Asymptotic crack tip fields}

We proceed to make use of the constitutive relations and the energy boundness constraint (36) to obtain the singularity power index $\beta$ in (24). Further, we will make use of the higher order equilibrium equation (10) to determine the singularity order of the Cauchy stress $\sigma_{i j}$. Note that (10) involves $\tau_{i j k, k}$ and the symmetric part of the curl of the defect stress. Thus, from (24), the analysis requires obtaining the solution for: (i) the curl of Nye's tensor, which is obtained from the curl of the plastic distortion, (ii) the plastic strain gradients, and (iii) the Laplacian of the plastic strain. Consider an incompressible solid where the asymptotic solution for $\gamma_{i j}^{p}$ is given by (24); in a polar coordinate system, the individual components of the plastic distortion tensor read,

$$
\gamma_{r r}^{p}=-\gamma_{\theta \theta}^{p} \sim r^{\beta} f_{1}(\theta) ; \quad \gamma_{r \theta}^{p} \sim r^{\beta} f_{2}(\theta) ; \quad \gamma_{\theta r}^{p} \sim r^{\beta} f_{3}(\theta)
$$

Accordingly, the solution for the plastic strain and plastic spin fields is of the following form,

$$
\varepsilon_{r r}^{p}=-\varepsilon_{\theta \theta}^{p} \sim r^{\beta} f_{1}(\theta) ; \quad \varepsilon_{r \theta}^{p} \sim r^{\beta} f_{4}(\theta) ; \quad \vartheta_{r \theta}^{p} \sim r^{\beta} f_{5}(\theta)
$$

And the relevant components of the plastic strain gradient and the Lapla- 
cian of the plastic strain readily follow,

$$
\begin{aligned}
& \varepsilon_{r r, r}^{p}=\frac{\partial \varepsilon_{r r}^{p}}{\partial r}=\beta r^{\beta-1} f_{1}(\theta) ; \quad \varepsilon_{r r, \theta}^{p}=\frac{1}{r}\left(\frac{\partial \varepsilon_{r r}^{p}}{\partial \theta}-2 \varepsilon_{r \theta}^{p}\right)=r^{\beta-1}\left(f_{1}^{\prime}(\theta)-2 f_{4}(\theta)\right) \\
& \varepsilon_{r \theta, r}^{p}=\frac{\partial \varepsilon_{r \theta}^{p}}{\partial r}=\beta r^{\beta-1} f_{4}(\theta) ; \quad \varepsilon_{r \theta, \theta}^{p}=\frac{1}{r}\left(\frac{\partial \varepsilon_{r \theta}^{p}}{\partial \theta}+2 \varepsilon_{r r}^{p}\right)=r^{\beta-1}\left(f_{4}^{\prime}(\theta)+2 f_{1}(\theta)\right) \\
& \varepsilon_{r r, k k}^{p}=\frac{\partial^{2} \varepsilon_{r r}^{p}}{\partial r^{2}}+\frac{1}{r} \frac{\partial \varepsilon_{r r}^{p}}{\partial r}+\frac{1}{r^{2}} \frac{\partial^{2} \varepsilon_{r r}^{p}}{\partial \theta^{2}}-\frac{4}{r^{2}}\left(\varepsilon_{r r}^{p}+\frac{\partial \varepsilon_{r \theta}^{p}}{\partial \theta}\right) \\
&=r^{\beta-2}\left[f_{1}(\theta)\left(\beta^{2}-4\right)+f_{1}^{\prime \prime}(\theta)+4 f_{4}^{\prime}(\theta)\right] \\
& \varepsilon_{r \theta, k k}^{p}=\frac{\partial^{2} \varepsilon_{r \theta}^{p}}{\partial r^{2}}+\frac{1}{r} \frac{\partial \varepsilon_{r \theta}^{p}}{\partial r}+\frac{1}{r^{2}} \frac{\partial^{2} \varepsilon_{r \theta}^{p}}{\partial \theta^{2}}+\frac{4}{r^{2}}\left(\frac{\partial \varepsilon_{r r}^{p}}{\partial \theta}-\varepsilon_{r \theta}^{p}\right) \\
&=r^{\beta-2}\left[f_{4}(\theta)\left(\beta^{2}-4\right)+f_{4}^{\prime \prime}(\theta)+4 f_{1}^{\prime}(\theta)\right]
\end{aligned}
$$

Furthermore, the relevant components of Nye's tensor in polar coordinates read

$$
\begin{gathered}
\alpha_{r z}=\left(\operatorname{curl} \gamma_{i j}^{p}\right)_{r z}=\frac{\partial \gamma_{r \theta}^{p}}{\partial r}-\frac{1}{r}\left[\frac{\partial \gamma_{r r}^{p}}{\partial \theta}-\left(\gamma_{r \theta}^{p}+\gamma_{\theta r}^{p}\right)\right] \\
=r^{\beta-1}\left[f_{2}(\theta)(\beta+1)-f_{1}^{\prime}(\theta)+f_{3}(\theta)\right] \\
\alpha_{\theta z}=\left(\operatorname{curl} \gamma_{i j}^{p}\right)_{\theta z}=\frac{\partial \gamma_{\theta \theta}^{p}}{\partial r}-\frac{1}{r}\left(\frac{\partial \gamma_{r \theta}^{p}}{\partial \theta}+2 \gamma_{r r}^{p}\right) \\
=r^{\beta-1}\left[f_{1}(\theta)(-2-\beta)-f_{2}^{\prime}(\theta)\right]
\end{gathered}
$$

Finally, the components related to the curl of the defect stress, $\xi_{i j}=$ $\operatorname{curl}\left(\mu L_{E}^{2} \operatorname{curl} \gamma_{i j}^{p}\right)=\eta_{i j}+\varphi_{i j}$, are obtained as 


$$
\begin{aligned}
\xi_{r r}= & \frac{1}{r}\left\{\frac{\partial\left[\frac{\partial \gamma_{r \theta}^{p}}{\partial r}-\frac{1}{r}\left(\frac{\partial \gamma_{r r}^{p}}{\partial \theta}-\gamma_{r \theta}^{p}-\gamma_{\theta r}^{p}\right)\right]}{\partial \theta}-\frac{\partial \gamma_{\theta \theta}^{p}}{\partial r}+\frac{1}{r}\left(\frac{\partial \gamma_{r \theta}^{p}}{\partial \theta}+2 \gamma_{r r}^{p}\right)\right\} \\
= & r^{\beta-2}\left[f_{1}(\theta)(2+\beta)+f_{2}^{\prime}(\theta)(2+\beta)-f_{1}^{\prime \prime}(\theta)+f_{3}^{\prime}(\theta)\right] \\
\xi_{r \theta}=- & \frac{\partial\left[\frac{\partial \gamma_{r \theta}^{p}}{\partial r}-\frac{1}{r}\left(\frac{\partial \gamma_{r r}^{p}}{\partial \theta}-\gamma_{r \theta}^{p}-\gamma_{\theta r}^{p}\right)\right]}{\partial r}=r^{\beta-2}(1-\beta)\left[f_{2}(\theta)(1+\beta)-f_{1}^{\prime}(\theta)+f_{3}(\theta)\right] \\
\xi_{\theta r}= & \frac{1}{r}\left\{\frac{\partial\left[\frac{\partial \gamma_{\theta \theta}^{p}}{\partial r}-\frac{1}{r}\left(\frac{\partial \gamma_{r \theta}^{p}}{\partial \theta}+2 \gamma_{r r}^{p}\right)\right]}{\partial \theta}+\frac{\partial \gamma_{r \theta}^{p}}{\partial r}-\frac{1}{r}\left(\frac{\partial \gamma_{r r}^{p}}{\partial \theta}-\gamma_{r \theta}^{p}-\gamma_{\theta r}^{p}\right)\right\} \\
= & r^{\beta-2}\left[f_{1}^{\prime}(\theta)(-3-\beta)+f_{2}(\theta)(\beta+1)-f_{2}^{\prime \prime}(\theta)+f_{3}(\theta)\right] \\
\xi_{\theta \theta}= & -\frac{\partial\left[\frac{\partial \gamma_{\theta \theta}^{p}}{\partial r}-\frac{1}{r}\left(\frac{\partial \gamma_{r \theta}^{p}}{\partial \theta}+2 \gamma_{r r}^{p}\right)\right]}{\partial r}=r^{\beta-2}(1-\beta)\left[f_{1}(\theta)(-2-\beta)-f_{2}^{\prime}(\theta)\right]
\end{aligned}
$$

We proceed to determine the index of the singularity, $\beta$, neglecting the angular functions. Thus, we estimate the singular order of the elastic strain energy density $\Psi^{e}$, the defect energy $\Phi$ and the dissipation potential $\varphi$ and take into consideration that the energy released at the crack tip must be finite, see Section 3.2 . We consider the general case, $\left(L_{D} \neq 0, L_{E} \neq 0\right)$, and particularize later.

The gradient term is more singular and dominates the asymptotic behaviour of the generalized plastic strain $E^{p}$, see (29); accordingly,

$$
E^{p} \sim L_{D} \varepsilon_{i j k}^{p} \sim r^{\beta-1}
$$

And its work conjugate stress reads,

$$
\Sigma=\Sigma_{0}\left(E^{p}\right)^{N} \sim r^{N(\beta-1)}
$$


Consequently, the asymptotic behaviour of the dissipative stresses associated with the primal kinematic variable $\varepsilon_{i j}^{p}$ is given by,

$$
q_{i j}=\frac{2}{3} \frac{\Sigma}{E^{p}} \varepsilon_{i j}^{p} \sim r^{N+\beta} ; \quad \tau_{i j k}=\frac{2}{3} L_{D}^{2} \frac{\Sigma}{E^{p}} \varepsilon_{i j, k}^{p} \sim r^{N+\beta-1}
$$

Now consider (43)-(44); the asymptotic behaviour of the energetic defect stress reads,

$$
\zeta_{i j}=\mu L_{E}^{2} \alpha_{i j}=\mu L_{E}^{2} \epsilon_{j k l} \gamma_{i l, k}^{p} \sim r^{\beta-1}
$$

Finally, the asymptotic behaviour of the Cauchy stress is obtained from the higher order equilibrium equation (10), which involves the Laplacian of the plastic strains via the dissipative term $\tau_{i j k, k}$ and the curl of the defect stress via the energetic term $\eta_{i j}^{\prime}$. Both terms are more singular than $q_{i j}$ and have in fact the same singularity order: $r^{\beta-2}$ - see (41)-(42) and (45)-(48). Hence,

$$
\sigma_{i j}^{\prime}=q_{i j}-\tau_{i j k, k}+\eta_{i j}^{\prime} \sim r^{\beta-2}
$$

In other words, the singularity exhibited by the crack tip stresses will be the same if only energetic higher order terms are present $\left(L_{E} \neq 0, L_{D}=0\right)$ and if only dissipative higher order terms are present $\left(L_{D} \neq 0, L_{E}=0\right)$. The use of Nye's tensor as primal higher order kinematic variable leads to identical asymptotic crack tip behaviour relative to the choice of a defect energy with the plastic strain tensor as primal variable. In all cases a quadratic form of the defect energy is assumed; interestingly, less-than-quadratic defect energies will have important implications in fracture problems: changing the nature of the stress singularity (if $L_{D}=0$ ) or making energetic contributions negligible relative to their dissipative counterparts (if $L_{D} \neq 0$ ). 
Consider now the relevant energy quantities. The elastic strains will have the same asymptotic behaviour as the Cauchy stresses, and consequently:

$$
\Psi^{e}=\frac{1}{2} C_{i j k l} \varepsilon_{i j}^{e} \varepsilon_{k l}^{e} \sim r^{2(\beta-2)}
$$

While the defect energy and dissipation potential vary as,

$$
\begin{gathered}
\Phi=\frac{1}{2} \mu L_{E}^{2} \alpha_{i j} \alpha_{i j} \sim r^{2(\beta-1)} \\
\varphi=\frac{\Sigma E^{p}}{N+1} \sim r^{(N+1)(\beta-1)}
\end{gathered}
$$

Therefore, $\Psi^{e}$ is the most singular contribution and will dominate the energy released in the vicinity of the crack tip - see (36). Since the total strain energy density must scale as $\sim J / r$ to give a finite energy release rate at the crack tip, we conclude that the singularity index $\beta$ must be equal to 3/2. The implications of this finding are the following:

- The elastic energy dominates as $r \rightarrow 0$ and the plastic field is not sufficiently singular to give any contribution to the energy release rate. If the plastic energy terms, $\Phi$ or $\varphi$, were to behave asymptotically as $J / r, \beta$ would be equal to 1 (for $N=0$ ) or smaller (if $N>0$ ) and the energy release rate at the crack tip would be unbounded $\left(\Psi^{e} \sim w \sim r^{-2}\right)$.

- Crack tip stresses follow the linear elastic $r^{-1 / 2}$ singularity, revealing the existence of an inner elastic $K$-field that is reminiscent of a dislocationfree zone.

- The plastic strain field $\varepsilon_{i j}^{p}$ tends to zero as the crack is approached. Crack tip asymptotic analyses for distortion gradient plasticity, and 
similar classes of gradient theories, should not be built on the assumption that plastic strains dominate elastic strains, as done for conventional plasticity (HRR field [52, 53]) and previous studies in strain gradient plasticity [54 56].

We proceed to corroborate these findings with detailed finite element analysis, as well as exploring other interesting features of distortion gradient plasticity predictions in fracture problems.

\section{Numerical formulation and solution procedure}

The flow theory of distortion gradient plasticity, described in Section 2, is implemented in a robust, backward Euler finite element framework. This is largely facilitated by the definition of a new viscoplastic potential, able to model both rate-dependent and rate-independent behaviour, by extending the work of Panteghini and Bardella [34].

\subsection{Viscoplastic law}

Gradient plasticity theories are commonly implemented within a ratedependent setting, taking advantage of its well-known computational capabilities and circumventing complications associated with identifying active

plastic zones in the corresponding time independent model [57, 58]. In the context of rate-dependent gradient plasticity models, an effective flow resistance $\Sigma$ is defined,

$$
\Sigma\left(\dot{E}^{p}, E^{p}\right)=\sigma_{F}\left(E^{p}\right) V\left(\dot{E}^{p}\right)
$$

which is work conjugated to the gradient-enhanced effective plastic flow rate $\dot{E}^{p}$. Here, $\sigma_{F}$ is the current flow stress, which depends on the initial yield 
stress $\sigma_{Y}$ and the hardening law. Several viscoplastic laws have been proposed in the literature; the most exploited one is arguably the following, (see, e.g., [32, 59]])

$$
\mathscr{V}\left(\dot{E}^{p}, E^{p}\right)=\frac{\sigma_{F}\left(E^{p}\right) \dot{\varepsilon}_{0}}{m+1}\left(\frac{\dot{E}^{p}}{\dot{\varepsilon}_{0}}\right)^{m+1}
$$

so that

$$
\Sigma\left(\dot{E}^{p}, E^{p}\right)=\sigma_{F} V\left(\dot{E}^{p}\right)=\sigma_{F}\left(E^{p}\right)\left(\frac{\dot{E}^{p}}{\dot{\varepsilon}_{0}}\right)^{m}
$$

with $m$ being the material rate sensitivity exponent, $\dot{\varepsilon}_{0}$ the reference strain rate and $V\left(\dot{E}^{p}\right)$ the viscoplastic function. However, under this choice the initial tangent is infinite and the derivative $\partial \Sigma / \partial \dot{E}^{p}$ tends to infinity if $\dot{E}^{p} \rightarrow$ 0, making the finite element system ill-conditioned for small values of $\dot{E}^{p}$. To overcome these numerical issues, Panteghini and Bardella [33] proposed the following viscoplastic function,

$$
V\left(\dot{E}^{p}\right)= \begin{cases}\frac{\dot{E}^{p}}{2 \dot{\varepsilon}_{0}} & \text { if } \dot{E}^{p} / \dot{\varepsilon}_{0} \leq 1 \\ 1-\frac{\dot{\varepsilon}_{0}}{2 \dot{E}^{p}} & \text { if } \dot{E}^{p} / \dot{\varepsilon}_{0}>1\end{cases}
$$

In this way, the contribution of $\partial \Sigma / \partial \dot{E}^{p}$ will remain bounded when $\dot{E}^{p} \rightarrow$ 0. This viscoplastic function is intended to reproduce the rate-independent limit in a robust manner, which is attained when $\dot{\varepsilon}_{0} \rightarrow 0$. We extend the work by Panteghini and Bardella [33] to develop a viscoplastic algorithm that can overcome the aforementioned numerical issues, and enables modelling both rate-dependent and rate-independent behaviour by recovering the well-known viscoplastic function $V\left(\dot{E}^{p}\right)=\left(\dot{E}^{p} / \dot{\varepsilon}_{0}\right)^{m}$. For this purpose, a threshold effective plastic strain rate is defined $\dot{E}_{*}^{p}$ such that the viscoplastic function 
reads,

$$
V\left(\dot{E}^{p}\right)= \begin{cases}\frac{\dot{E}^{p}}{\varpi \dot{\varepsilon}_{0}} & \text { if } \dot{E}^{p} m / \dot{E}_{\star}^{p} \leq 1 \\ \left(\frac{\dot{E}^{p}-\frac{1-m}{m} \dot{E}_{\star}^{p}}{\dot{\varepsilon}_{0}}\right)^{m} & \text { if } \dot{E}^{p} m / \dot{E}_{\star}^{p}>1\end{cases}
$$

where $\varpi$ is a small positive constant $(\varpi<<1)$. A smooth transition is obtained by computing the critical $\dot{E}_{*}^{p}$ from the relation between the derivatives,

$$
\dot{E}_{\star}^{p}=\dot{\varepsilon}_{0}\left(\frac{1}{\varpi m}\right)^{1 /(m-1)}
$$

and by offsetting the curve a distance $\dot{E}_{*}^{p}(1-m) / m$. This distance corresponds to the intersection between the abscissa axis and the tangent line at the critical point. In this way, we are able to reproduce a mechanical response that accurately follows the classic viscoplastic power law while providing a robust numerical framework. Representative curves for the aforementioned viscoplastic functions are shown in Fig. 1; the regularisation proposed here approximates the classic viscoplastic function very well, enabling it to reproduce the rate sensitivity of metals, while retaining the robustness of the proposal by Panteghini and Bardella [33]. 


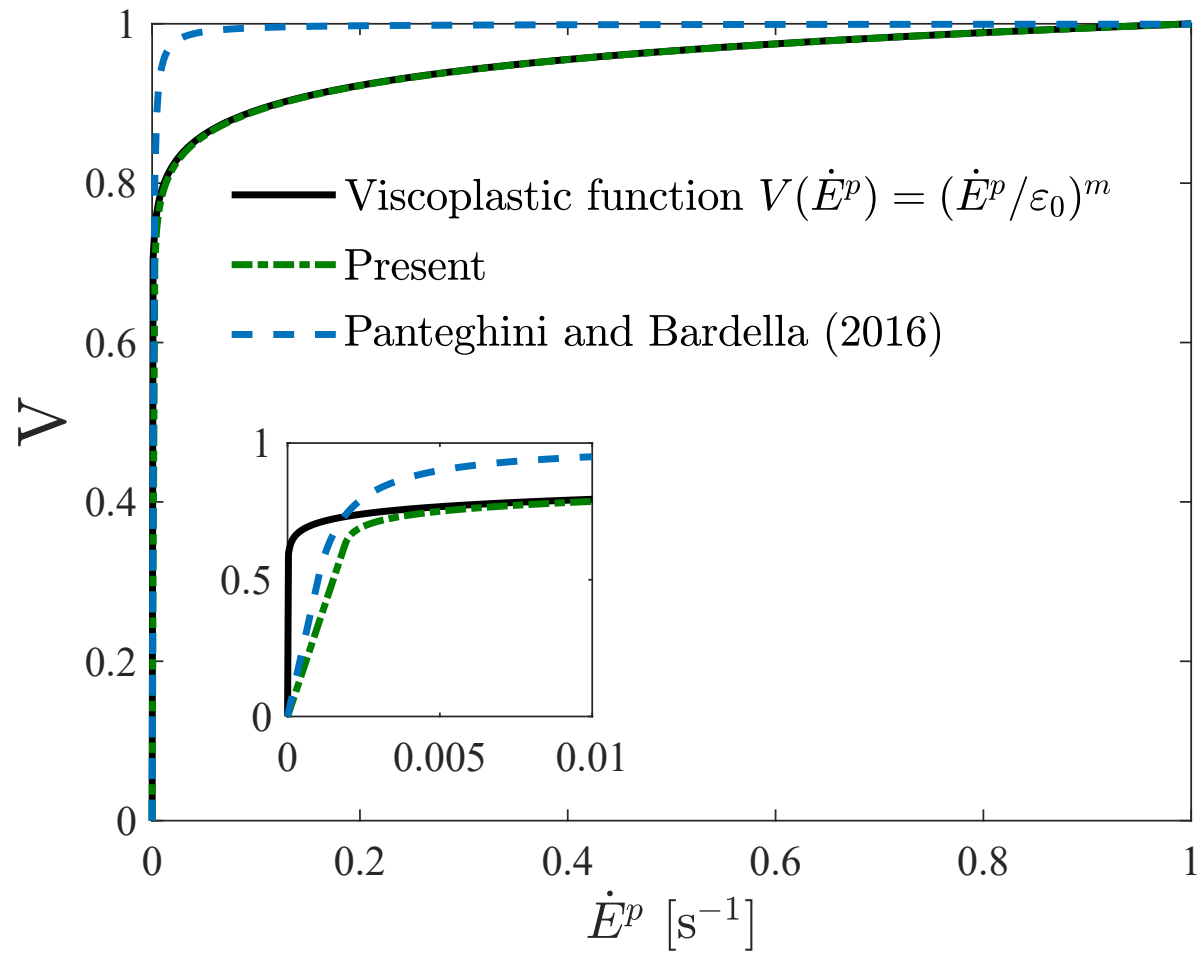

Figure 1: Comparison between the classic viscoplastic power law, the viscoplastic function presented and the one proposed by Panteghini and Bardella [33]. In the function by Panteghini and Bardella [33] the reference strain rate $\dot{\varepsilon}_{0}$ equals $10^{3} \mathrm{~s}^{-1}$, while in the other two cases a rate sensitivity exponent of $m=0.05$ and a reference strain rate $\dot{\varepsilon}_{0}=1$ are adopted; these choices pertain only to the present graph.

\subsection{Finite element discretisation}

The finite element framework takes displacements, plastic strains and plastic spin as the primary kinematic variables. Adopting symbolic and Voigt notation, the nodal variables for the displacement field $\hat{\boldsymbol{u}}$, the plastic strains $\hat{\boldsymbol{\varepsilon}}^{p}$, and the plastic spin $\hat{\vartheta}^{p}$ are interpolated as,

$$
\boldsymbol{u}=\sum_{n=1}^{k} \boldsymbol{N}_{n}^{\boldsymbol{u}} \hat{\boldsymbol{u}}_{n}, \quad \boldsymbol{\varepsilon}^{p}=\sum_{n=1}^{k} \boldsymbol{N}_{n}^{\varepsilon^{p}} \hat{\boldsymbol{\varepsilon}}_{n}^{p}, \quad \boldsymbol{\vartheta}^{p}=\sum_{n=1}^{k} \boldsymbol{N}_{n}^{\vartheta^{p}} \hat{\boldsymbol{\vartheta}}_{n}^{p}
$$


Here, $N_{n}$ denotes the shape function associated with node $n$, for a total number of nodes $k$. Similarly, the related gradient and curl-based quantities are discretised as

$$
\boldsymbol{\varepsilon}=\sum_{n=1}^{k} \boldsymbol{B}_{n}^{\boldsymbol{u}} \hat{\boldsymbol{u}}_{n}, \quad \nabla \boldsymbol{\varepsilon}^{p}=\sum_{n=1}^{k} \boldsymbol{B}_{n}^{\boldsymbol{\varepsilon}^{p}} \hat{\boldsymbol{\varepsilon}}_{n}^{p}, \quad \boldsymbol{\alpha}=\sum_{n=1}^{k}\left(\boldsymbol{M}_{n}^{\varepsilon^{p}} \hat{\boldsymbol{\varepsilon}}_{n}^{p}+\boldsymbol{M}_{n}^{\boldsymbol{\vartheta}^{p}} \hat{\boldsymbol{\vartheta}}_{n}^{p}\right)
$$

with the $\boldsymbol{B}$ and $\boldsymbol{M}$ matrices given explicitly in Appendix A. Accordingly, one can discretise the internal virtual work (7) as,

$$
\begin{aligned}
\delta W_{i}= & \int_{\Omega}\left\{\left(\boldsymbol{B}_{n}^{\boldsymbol{u}}\right)^{T} \boldsymbol{\sigma} \delta \hat{\boldsymbol{u}}_{n}+\left[\left(\boldsymbol{N}_{n}^{\vartheta^{p}}\right)^{T} \boldsymbol{\omega}+\left(\boldsymbol{M}_{n}^{\vartheta^{p}}\right)^{T} \boldsymbol{\zeta}\right] \delta \hat{\boldsymbol{\vartheta}}_{n}^{p}\right. \\
& \left.+\left[\left(\boldsymbol{N}_{n}^{\varepsilon^{p}}\right)^{T}(\boldsymbol{q}-\boldsymbol{\sigma})+\left(\boldsymbol{B}_{n}^{\boldsymbol{\varepsilon}^{p}}\right)^{T} \boldsymbol{\tau}+\left(\boldsymbol{M}_{n}^{\varepsilon^{p}}\right)^{T} \boldsymbol{\zeta}\right] \delta \hat{\boldsymbol{\varepsilon}}_{n}^{p}\right\} \mathrm{d} V
\end{aligned}
$$

Differentiating the internal virtual work with respect to the variation of the nodal variables provides the residuals for each kinematic variable as:

$$
\begin{gathered}
\boldsymbol{R}_{n}^{u}=\int_{\Omega}\left(\boldsymbol{B}_{n}^{u}\right)^{T} \boldsymbol{\sigma} \mathrm{d} V \\
\boldsymbol{R}_{n}^{\boldsymbol{\varepsilon}^{p}}=\int_{\Omega}\left[\left(\boldsymbol{N}_{n}^{\varepsilon^{p}}\right)^{T}(\boldsymbol{q}-\boldsymbol{\sigma})+\left(\boldsymbol{B}_{n}^{\boldsymbol{\varepsilon}^{p}}\right)^{T} \boldsymbol{\tau}+\left(\boldsymbol{M}_{n}^{\boldsymbol{\varepsilon}^{p}}\right)^{T} \boldsymbol{\zeta}\right] \mathrm{d} V \\
\boldsymbol{R}_{n}^{\vartheta^{p}}=\int_{\Omega}\left[\left(\boldsymbol{N}_{n}^{\vartheta^{p}}\right)^{T} \boldsymbol{\omega}+\left(\boldsymbol{M}_{n}^{\boldsymbol{\vartheta}^{p}}\right)^{T} \boldsymbol{\zeta}\right] \mathrm{d} V
\end{gathered}
$$

The components of the consistent tangent stiffness matrices $\boldsymbol{K}_{n m}$ are obtained by considering the constitutive relations and differentiating the residuals with respect to the incremental nodal variables. Details are given in Appendix A. The non-linear system of equations is solved iteratively from time step $t$ to $(t+\Delta t)$ using the Newton-Raphson method,

$$
\left[\begin{array}{c}
\boldsymbol{u} \\
\boldsymbol{\varepsilon}^{p} \\
\boldsymbol{\vartheta}^{p}
\end{array}\right]_{t+\Delta t}=\left[\begin{array}{c}
\boldsymbol{u} \\
\boldsymbol{\varepsilon}^{p} \\
\boldsymbol{\vartheta}^{p}
\end{array}\right]_{t}-\left[\begin{array}{ccc}
\boldsymbol{K}^{u, u} & \boldsymbol{K}^{u, \varepsilon^{p}} & \mathbf{0} \\
\boldsymbol{K}^{\varepsilon^{p}, u} & \boldsymbol{K}^{\varepsilon^{p}, \varepsilon^{p}} & \boldsymbol{K}^{\varepsilon^{p}, \vartheta^{p}} \\
\mathbf{0} & \boldsymbol{K}^{\vartheta^{p}, \varepsilon^{p}} & \boldsymbol{K}^{\vartheta^{p}, \vartheta^{p}}
\end{array}\right]_{t}^{-1}\left[\begin{array}{c}
\boldsymbol{R}^{\boldsymbol{u}} \\
\boldsymbol{R}^{\varepsilon^{p}} \\
\boldsymbol{R}^{\vartheta}
\end{array}\right]_{t}
$$


The present backward Euler time integration scheme follows the work by Panteghini and Bardella [33]; see Ref. [32] for a forward Euler based implementation. The finite element framework is implemented into the commercial package ABAQUS by means of a user element (UEL) subroutine.

\section{Finite Element results}

The numerical model described in Section 4 is employed to gain insight into the fracture behaviour of distortion gradient plasticity solids. First, the analysis will be conducted with a boundary layer configuration, under

small scale yielding conditions (Section 5.1). Irrotational plastic flow and mode I fracture will be assumed first, to verify the findings of the asymptotic analysis and assess the role of Nye's tensor. Mixed mode conditions are then considered to address the role of the plastic spin. Finally, fracture along a bi-material interface is investigated by reproducing the four-point bending experiments by Korn et al. [49] with suitable higher order boundary conditions (Section 5.2).

\subsection{Small scale yielding}

A remote $K$-field is prescribed by means of the so-called boundary layer formulation, see Fig. 2. Plane strain conditions are assumed. Consider both a polar coordinate system $(r, \theta)$ and a Cartesian coordinate system $(x, y)$ centred at the crack tip, with the crack plane along the negative $x$-axis. The outer $K$ field is imposed by prescribing the nodal displacements in the outer periphery of the mesh as,

$$
u_{x}=\frac{1+\nu}{E} \sqrt{\frac{r}{2 \pi}}\left[K_{I}(3-4 \nu-\cos \theta) \cos \left(\frac{\theta}{2}\right)+K_{I I}(5-4 \nu+\cos \theta) \sin \left(\frac{\theta}{2}\right)\right]
$$




$$
u_{y}=\frac{1+\nu}{E} \sqrt{\frac{r}{2 \pi}}\left[K_{I}(3-4 \nu-\cos \theta) \sin \left(\frac{\theta}{2}\right)+K_{I I}(1-4 \nu+\cos \theta) \cos \left(\frac{\theta}{2}\right)\right]
$$

where $E$ is Young's modulus, $\nu$ is Poisson's ratio and $K_{I}$ and $K_{I I}$ respectively denote the mode I and mode II stress intensity factors. Upon exploiting the symmetry about the crack plane, only half of the finite element model is analysed. After a mesh sensitivity study, the domain is discretised with 11,392 quadrilateral quadratic elements with full integration. As shown in Fig. 2, the mesh is progressively refined towards the crack tip to resolve the material strain gradient length $\ell$. From the outer $K$-field, a representative length of the plastic zone can be defined as,

$$
R_{p}=\frac{1}{3 \pi}\left(\frac{K}{\sigma_{Y}}\right)^{2}
$$

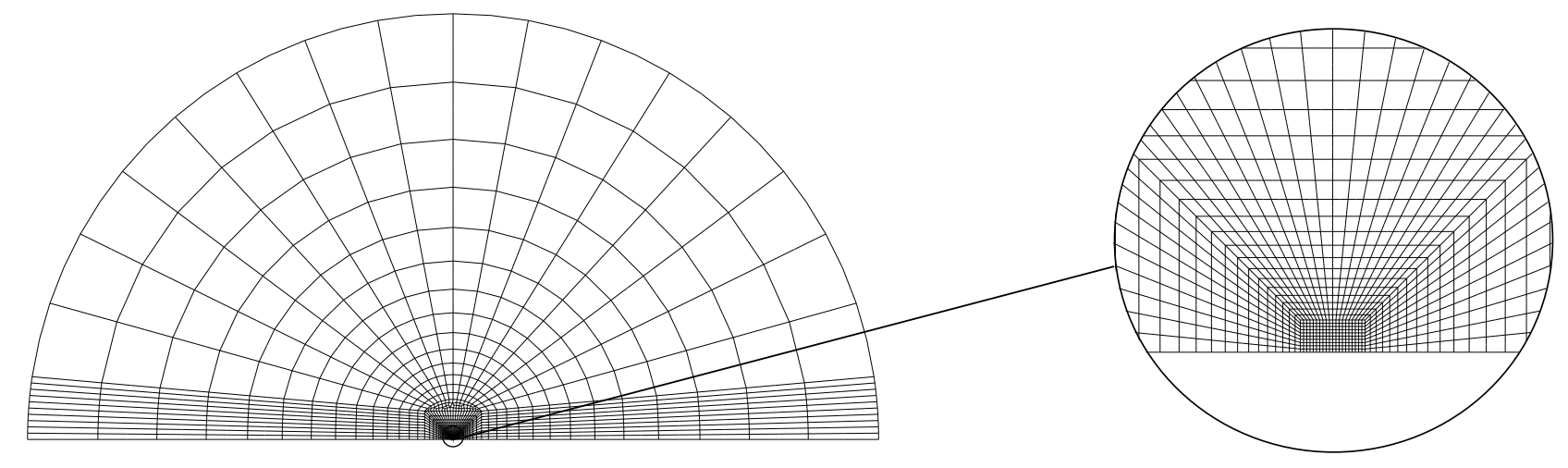

Figure 2: Boundary layer formulation. Schematic and detailed view of the finite element mesh.

We assume that the material obeys the following isotropic power-law 
hardening rule:

$$
\sigma_{F}=\sigma_{Y}\left(1+\frac{E E^{p}}{\sigma_{Y}}\right)^{N}
$$

with the current flow stress, $\sigma_{F}$, being related to the gradient-enhanced effective plastic flow rate through the viscoplastic function - see (57). The viscoplastic parameters are chosen to model the rate-independent limit. Specifically, following Ref. [41], we define the following dimensionless constant:

$$
c=\frac{\dot{K} \varepsilon_{Y}}{K \dot{\varepsilon}_{0}}
$$

where $\varepsilon_{Y}=\sigma_{Y} / E$ is the yield strain, and make suitable choices for $c$ and $m$. By comparing with the results obtained with rate-independent $J_{2}$ plasticity and the viscoplastic function by Panteghini and Bardella [33] (with $\dot{\varepsilon}_{0} \rightarrow$ 0 ), we find that $c=0.25$ and $m=0.005$ accurately approximate the rateindependent limit. Throughout Section 5.1, material properties are assumed to be $\sigma_{Y} / E=0.003, \nu=0.3$ and $N=0.1$. We investigate the influence of $\chi$, the parameter that governs dissipation due to the plastic spin, and the ratio $\ell / R_{p}$, where $\ell$ is a reference length scale $L_{E}=L_{D}=\ell$. In addition, insight is gained into the role of the individual energetic $L_{E}$ and dissipative $L_{D}$ length scales.

\subsubsection{Asymptotic behaviour under Mode I fracture}

We proceed to verify the analytical findings of the asymptotic study in Section 3. Assume pure mode I conditions $\left(K_{I I}=0\right)$ and irrotational plastic flow $(\chi \rightarrow \infty)$. The tensile stress distribution ahead of the crack tip is shown in log-log scale in Fig. [3 for selected values of $\ell / R_{p}$. The finite element results confirm the analytical findings; for all $\ell / R_{p}>0$ values an elastic stress state exists close to the crack tip, where $\sigma_{y y}$ scales as $r^{-1 / 2}$. 


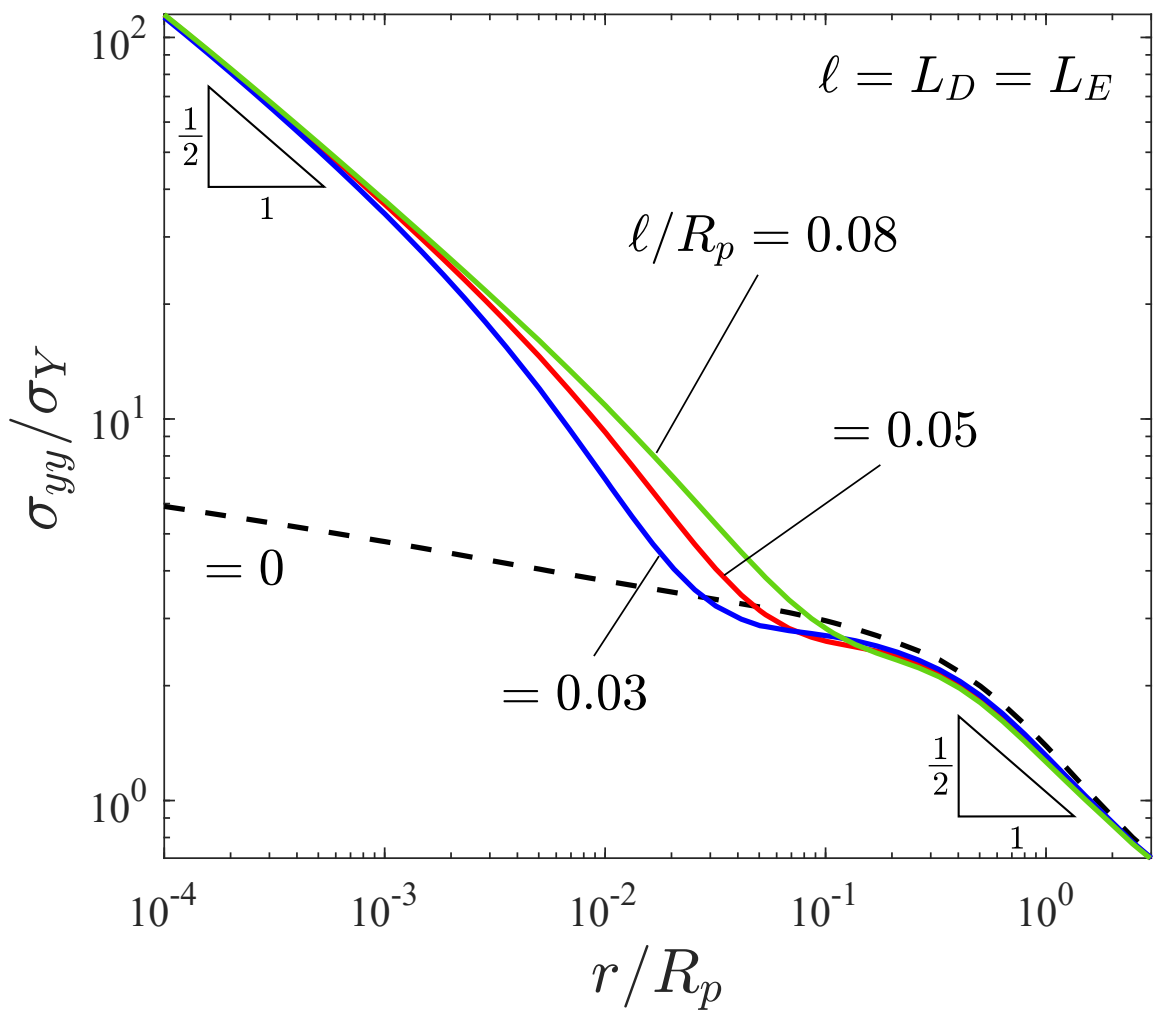

Figure 3: Tensile stress distribution ahead of the crack tip for selected values of $\ell / R_{p}$. Material properties: $\sigma_{Y} / E=0.003, \nu=0.3, N=0.1$, and $\chi \rightarrow \infty$.

Several distinct regions are seen in Fig. 3. Far away from the crack tip, the stress field is elastic and exhibits the linear elastic singularity $r^{-1 / 2}$. As the crack tip is approached a plastic region arises, where the stresses follow the HRR field of conventional $J_{2}$ plasticity [52, 53], with $\sigma_{y y}$ scaling as $r^{-N /(N+1)}$. This plastic region is reached at $r \approx 0.5 R_{p}$, as the Irwin approximation for the plastic zone length (72) overestimates its size for strain hardening materials [60]. At approximately $r \leq \ell$, strain gradient hardening starts to play a role and a stress elevation is seen relative to the classic plasticity predic- 
tion (black dashed line, $\ell / R_{p}=0$ ). The size of the domain where gradient plasticity and conventional plasticity predictions deviate from each other is governed by $\ell / R_{p}$. Also, in the region $0.001 R_{p} \leq r \leq 0.1 R_{p}$ the degree of stress elevation relative to conventional plasticity increases with $\ell / R_{p}$. However, in the vicinity of the crack tip $(r \approx 0.1 \ell$ or smaller $)$, all $\ell / R_{p}>0$ cases superimpose, with the stress exhibiting the singular behaviour of linear elasticity $\sigma_{y y} \sim r^{-1 / 2}$, as predicted in the analytical asymptotic study. Note that path independence of the $J$-integral (35) implies that the outer and inner elastic $K$ fields must be the same; i.e., the inner $K$ field is identical for all $\ell / R_{p}>0$ values and corresponds to the one predicted by linear elasticity. Further insight into this elastic crack tip region is gained by plotting the ratio between the plastic strain and the elastic strain $\varepsilon_{y y}^{p} / \varepsilon_{y y}^{e}$; results are shown in Fig. 4 for conventional plasticity $\left(\ell / R_{p}=0\right)$ and gradient plasticity $\left(\ell / R_{p}=0.05\right)$. 


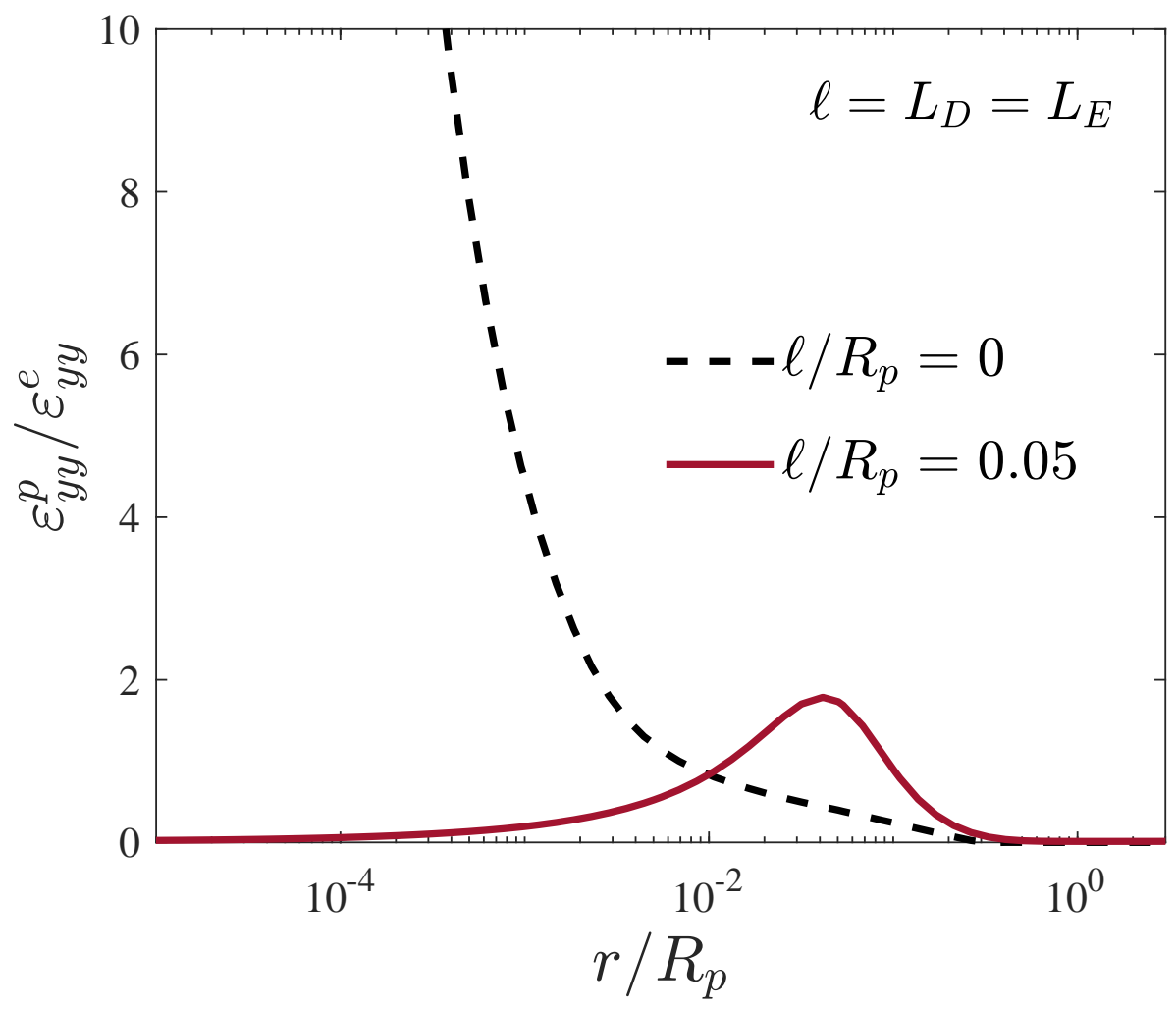

Figure 4: Distribution ahead of the crack tip of the ratio between the tensile plastic and elastic strains $\varepsilon_{y y}^{p} / \varepsilon_{y y}^{e}$ for both conventional plasticity $\left(\ell / R_{p}=0\right)$ and gradient plasticity $\left(\ell / R_{p}=0.05\right)$. Material properties: $\sigma_{Y} / E=0.003, \nu=0.3, N=0.1$, and $\chi \rightarrow \infty$.

As in Fig. 3, three regimes can be identified in Fig. 4. For both $\ell=0$ and $\ell>0$, far away from the crack tip the plastic strains are zero but eventually increase as $r$ becomes smaller than $R_{p}$. In conventional plasticity the plastic strains are singular and raise sharply as we approach the crack tip. However, when $\ell>0$ the ratio $\varepsilon_{y y}^{p} / \varepsilon_{y y}^{e}$ reaches a peak and then drops, with the elastic strains dominating when $r \rightarrow 0$. An elastic strain (and stress) state exists near the crack tip, where plastic strains are negligible. Thus, the assumption of a dominating plastic strain field as $r \rightarrow 0$ cannot be used to derive 
the asymptotic fields, as done in the context of conventional plasticity. This crack tip elastic core resembles the concept of a dislocation-free zone [61].

We proceed to assess the role of the individual energetic and dissipative higher order contributions. The crack tip stress distribution and the crack tip opening profile are respectively shown in Fig. 5a and Fig. 5b, Dissipative higher order effects dominate the crack tip response; the magnitude of $L_{E} / R_{p}$ has to be increased 50 times relative to $L_{D} / R_{p}$ to achieve a similar degree of crack tip stress elevation. Given that we are under nearly proportional loading, differences must be due to the constitutive choices for the energetic defect stress (18) and the dissipative higher order stress tensor $(22 \mathrm{c})$. In other words, the use of Nye's tensor as primal kinematic variable considerably reduces the local strengthening predicted ahead of a crack. The effect will likely be more profound if a less-than-quadratic defect energy is employed. Moreover, as it can be deduced from the analysis of Section 3 , less-than-quadratic defect energies will change the nature of the singularity - the crack tip stress state will no longer be elastic if $L_{D}=0$. For the present formulation, where the defect energy is quadratic, the asymptotic behaviour described by the purely energetic result is the same as in the purely dissipative case; as shown analytically, if $L_{E}>0$ or $L_{D}>0$ the stress field exhibits the elastic singularity $r^{-1 / 2}$ as $r \rightarrow 0$. It is important to note that, in both the analytical and numerical analyses, the plastic distortion field is assumed to be continuous. However, for the case $L_{D}=0$ (where gradient effects are due to Nye's tensor only), the theoretical framework is characterised by kinematic higher order boundary conditions that admit discontinuity in some 
components of the plastic distortion. Thus, the results reported for the case $L_{D}=0$ should be taken with care; an $H$ (curl) finite element framework, such as the one developed by Panteghini and Bardella [34], is needed to capture the discontinuities that might arise in Nye's tensor components. A very different outcome might be predicted if $\gamma_{i j}^{p}$ is allowed to be discontinuous and the solution localises. 


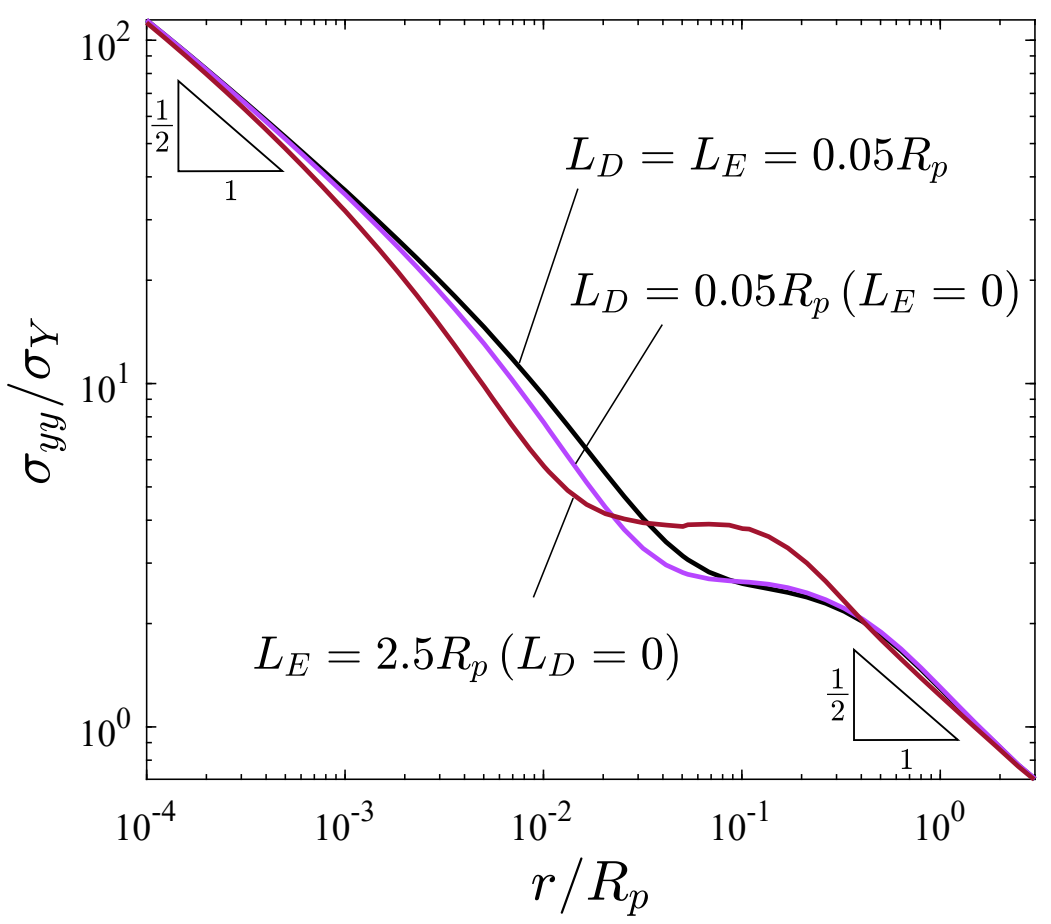

(a)

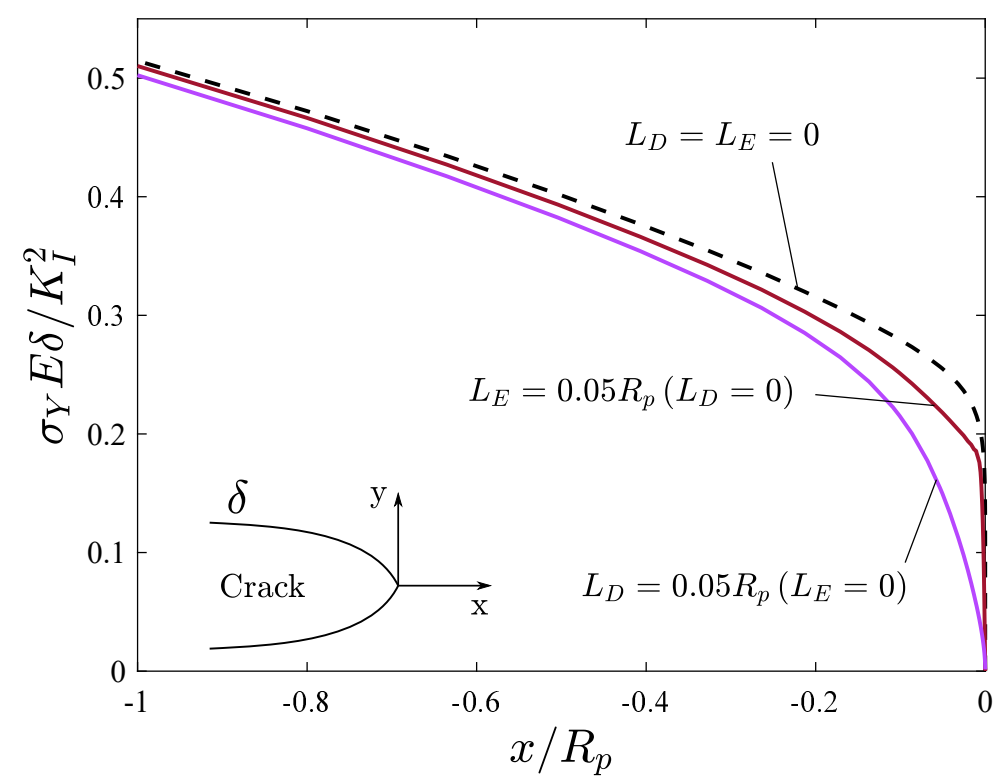

(b)

Figure 5: Influence of energetic and dissipative length scales: (a) tensile stress distribution ahead of the crack tip, and (b) crack opening profile. Material properties: $\sigma_{Y} / E=0.003$, $\nu=0.3, N=0.1$, and $\chi \rightarrow \infty$. 
As shown in Fig. 5b, the assumption of an equal magnitude for $L_{E}$ and $L_{D}$ leads to very different crack opening profiles. In the case of $L_{D}>0$ blunting is significantly reduced behind the crack tip. The crack profile also sharpens relative to the conventional plasticity prediction when $L_{E}>0\left(L_{D}=0\right)$ but to a much lesser extent. Far from the crack tip, the crack profile predictions for energetic gradient plasticity, dissipative gradient plasticity and conventional plasticity agree. Outside of the inner elastic core, the local strengthening predicted by Nye's tensor is much weaker than the one predicted by a gradient contribution based on the plastic strain gradient tensor. This is further explored in Fig. 6, where the gradient dominated zone $r_{D G P}$ is plotted as a function of the remote mode I load $K_{I}$. As in Ref. [38], we define $r_{D G P}$ to represent the length of the region ahead of the crack tip where the stress distribution significantly deviates from conventional plasticity: $\sigma_{D G P}>2 \sigma_{H R R}$. 


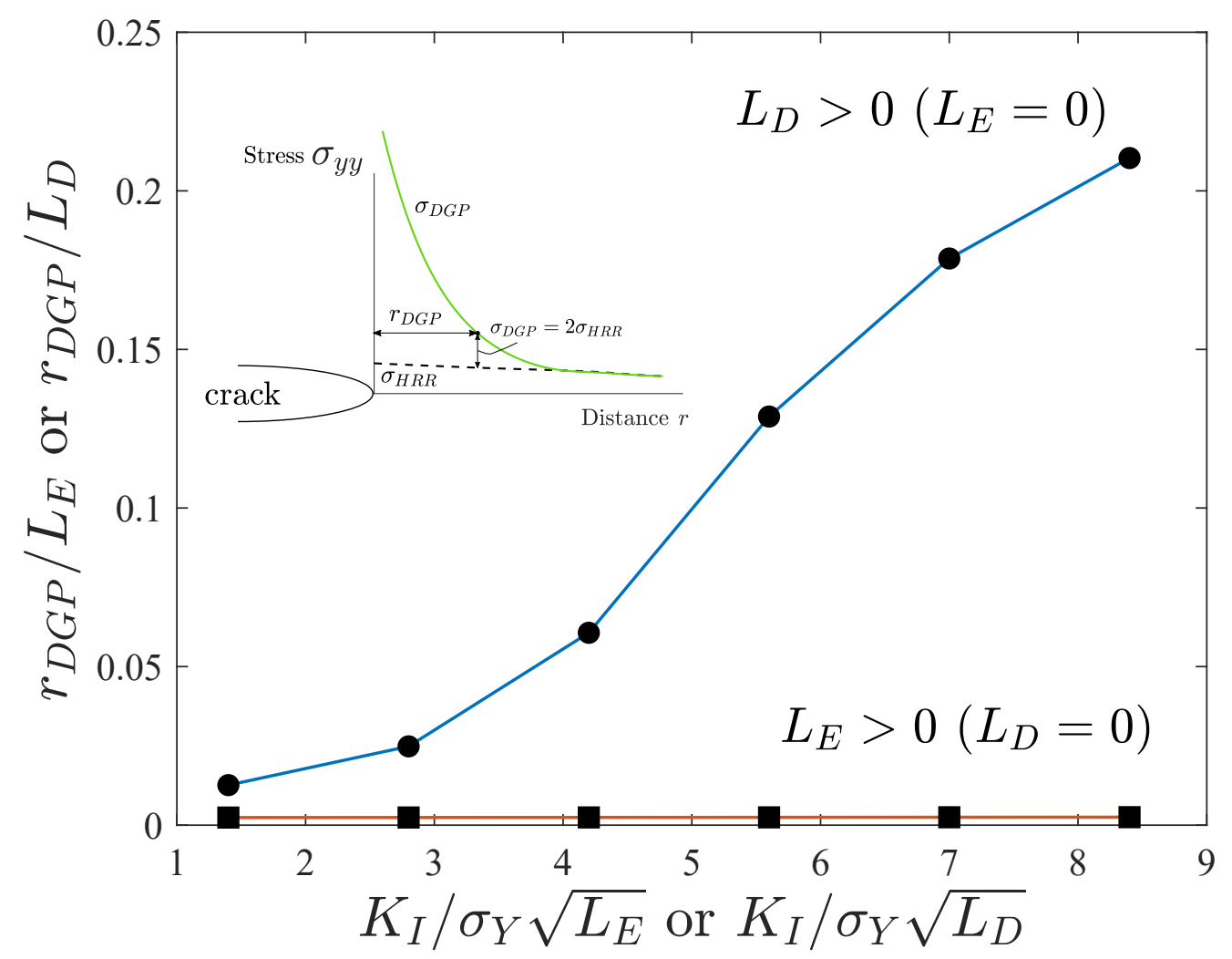

Figure 6: Distance ahead of the crack tip where gradient effects significantly elevate the stresses relative to conventional plasticity $\left(\sigma_{D G P}>2 \sigma_{H R R}\right)$. Material properties: $\sigma_{Y} / E=$ $0.003, \nu=0.3, N=0.1$, and $\chi \rightarrow \infty$.

The results shown in Fig. 6 reveal a minor sensitivity of $r_{D G P}$ with the remote load for the case $L_{E}>0\left(L_{D}=0\right)$. Tensile stresses are much higher than conventional plasticity in the elastic crack tip region but rapidly decay towards the conventional plasticity result farther away from the crack. Contrarily, in the case of $L_{D}>0$ the domain ahead of the crack where gradient effects significantly alter the stress distribution increases with the applied load. At the largest load level, the length of the stress elevation region is 
more than one order of magnitude larger if dissipative strengthening is accounted for. These differences are undoubtedly rooted in the choice of a free energy based on Nye's tensor. As shown in Ref. [39] for fracture and in Ref. [62] for bending, the dissipative contribution also outweighs the energetic counterpart when the defect energy is based on the plastic strain gradient tensor but differences are significantly smaller.

Insight into the role of Nye's tensor is further gained by plotting the distribution ahead of the crack tip of the relevant component, $\alpha_{y z}$ - see Fig. 7. The peak value of $\alpha_{y z}$ appears to saturate with an increasing remote load, reaching a maximum value on the order of $0.1 / L_{E}$. Given that $L_{E}$ is typically within the 1-10 $\mu \mathrm{m}$ range (see Table 1), the maximum value of $\alpha_{y z}$ is on the order of 0.01-0.1 $\mu \mathrm{m}^{-1}$, consistent with experimental observations [63]. 


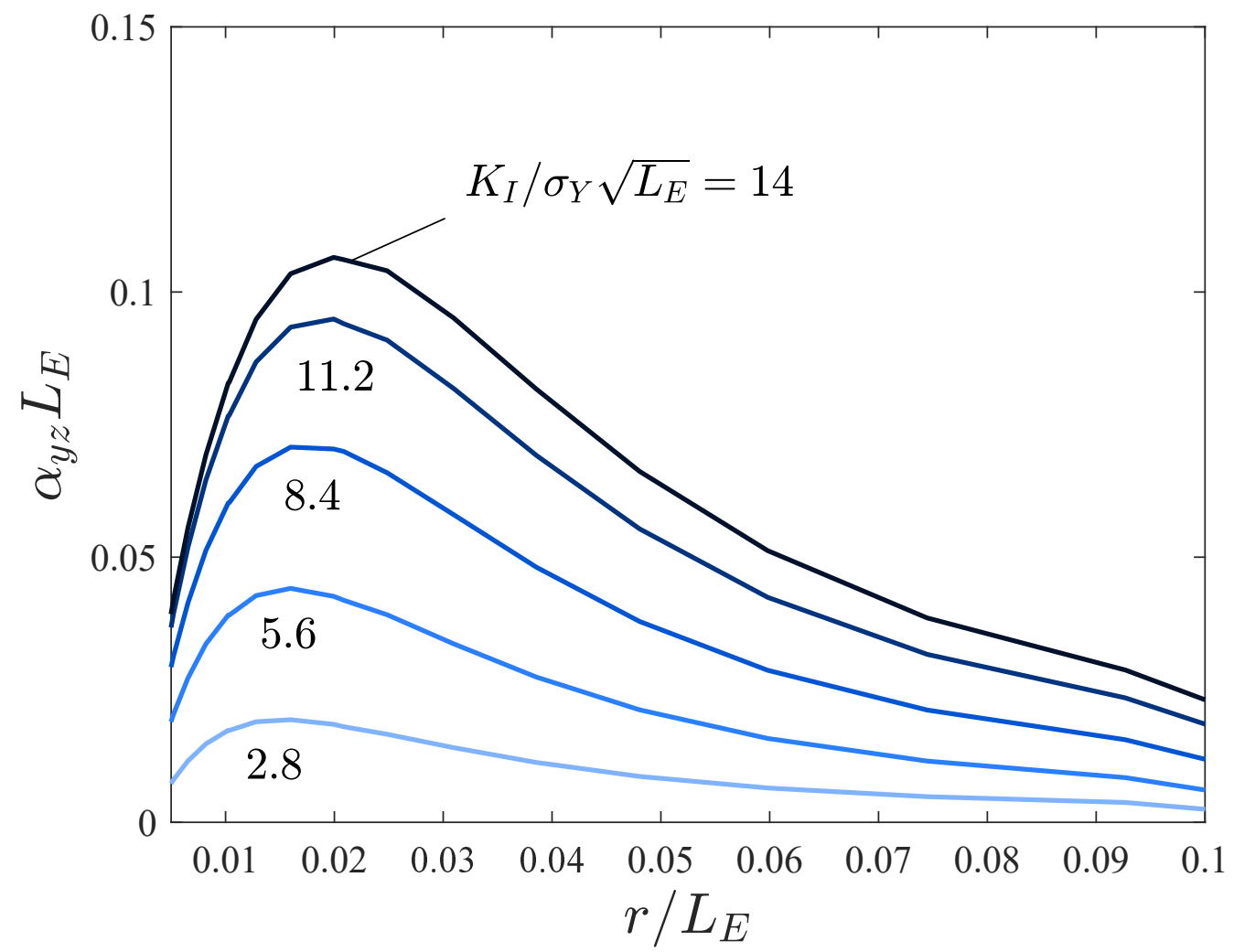

Figure 7: Nye's tensor component $\alpha_{y z}$ distribution ahead of the crack tip for selected values of the remote load and the case $L_{E}>0\left(L_{D}=0\right)$. Material properties: $\sigma_{Y} / E=0.003$, $\nu=0.3, N=0.1$, and $\chi \rightarrow \infty$.

The results shown in Figs. 5 [7 7 reveal that, beyond the elastic inner core, a curl-based defect energy requires larger $L_{E} / R_{p}$ values to have a similar impact on the stress distribution as gradient-based constitutive choices. This raises the following question: what are the values of $L_{E}$ that fit the outcome of micro-scale experiments for curl- and gradient-based models? To the best of 
the authors' knowledge this data does not exist, motivating future work 1

\subsubsection{Mixed-model fracture - the role of $\chi$}

We proceed to assess the role of $\chi$ and the plastic spin. For that, mixedmode fracture conditions are considered, where $K_{I}>0$ and $K_{I I}>0$. The degree of mode-mixity can be characterised by the following angle:

$$
\psi=\tan ^{-1}\left(\frac{K_{I I}}{K_{I}}\right)
$$

Note that, in the present model, the dissipation due to the plastic spin gradient is not accounted for. Accordingly, the plastic spin has no influence on the crack tip asymptotic behaviour; i.e., an elastic core exists, independently of the value of $\chi$. The plastic spin can play a role if we assume that the internal work is affected by different plastic rotations of two neighbouring macroscopic material points, as proposed by Bardella [27]. In the context of the original distortion gradient plasticity model [21], the influence of the plastic spin is limited to the stress elevation in the plastic region.

Crack tip stress fields are shown in Fig. 8 for $\psi=45^{\circ}$ and selected values of the parameter governing the dissipation due to the plastic spin, $\chi$. The choice $\chi=2 / 3$ makes the effective plastic flow rate (19) equal to the norm of the plastic distortion in the absence of higher order terms, while $\chi \rightarrow \infty$ reproduces the conditions of the theory by Gurtin and Anand [12] (that is, irrotational plastic flow). The results reveal a small influence of the plastic

\footnotetext{
${ }^{1}$ Bardella and Panteghini [19] obtained a good fit to the torsion experiments by Fleck et al. 2] with a curl-based model but employed a logarithmic defect energy.
} 
spin, with the stress level increasing with $\chi$. This agrees with the trends observed by Bardella [27] in the simple shear problem, where augmenting $\chi$ leads to additional material hardening.

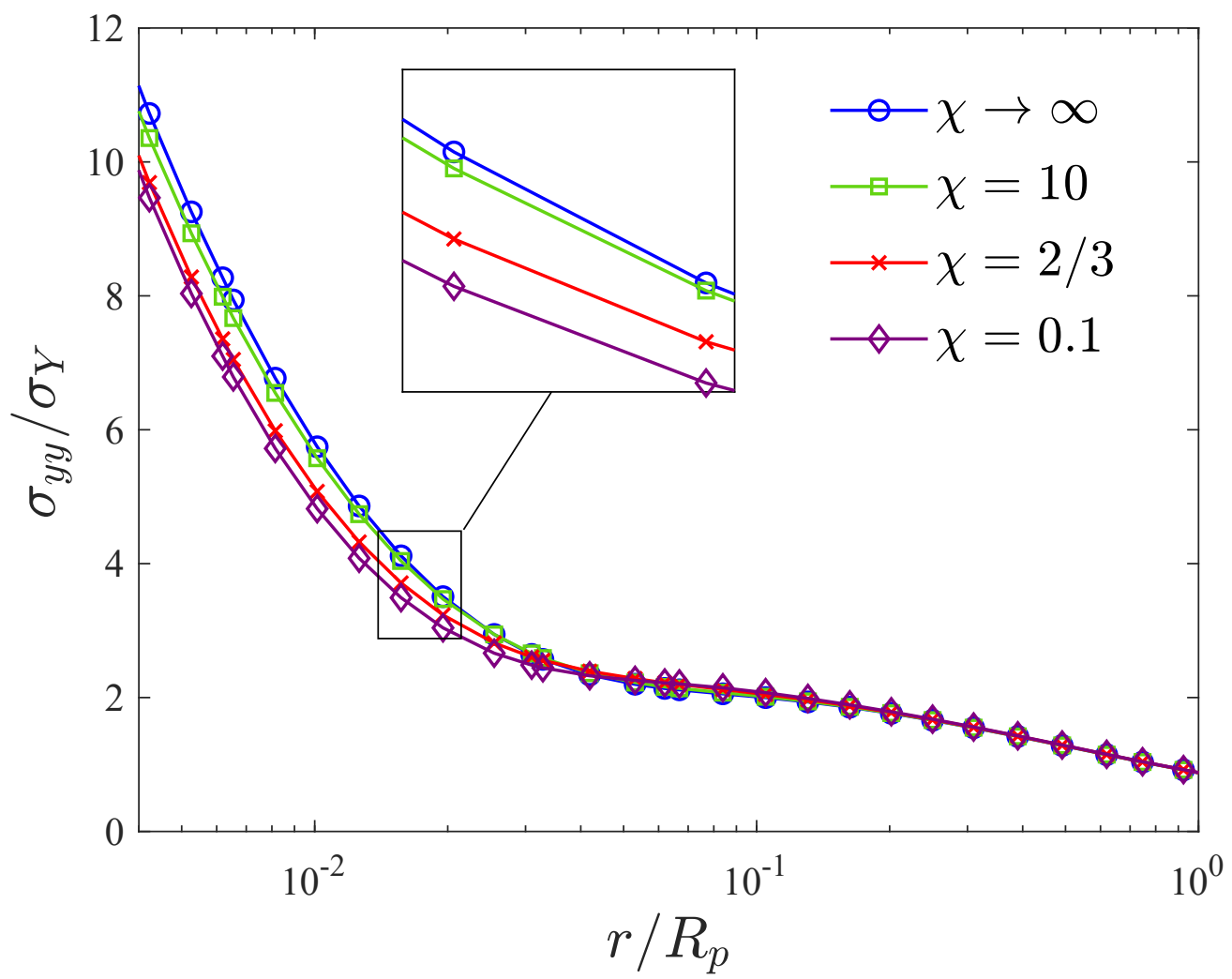

Figure 8: Tensile stress distribution ahead of the crack tip under mode-mix conditions, $\psi=45^{\circ}$, for selected values of $\chi$. Material properties: $\sigma_{Y} / E=0.003, \nu=0.3, N=0.1$, and $\ell / R_{p}=0.03$.

The role of the plastic spin in elevating crack tip stresses is further investigated by computing the stress elevation relative to conventional plasticity $\sigma_{D G P} / \sigma_{H R R}$ as a function of the degree of mode mixity, as given by the angle $\psi$. The results are shown in Fig. 9, It is found that the influence of the plas- 
tic spin increases with decreasing $\psi$, and that the stress elevation increases with increasing $\psi$.

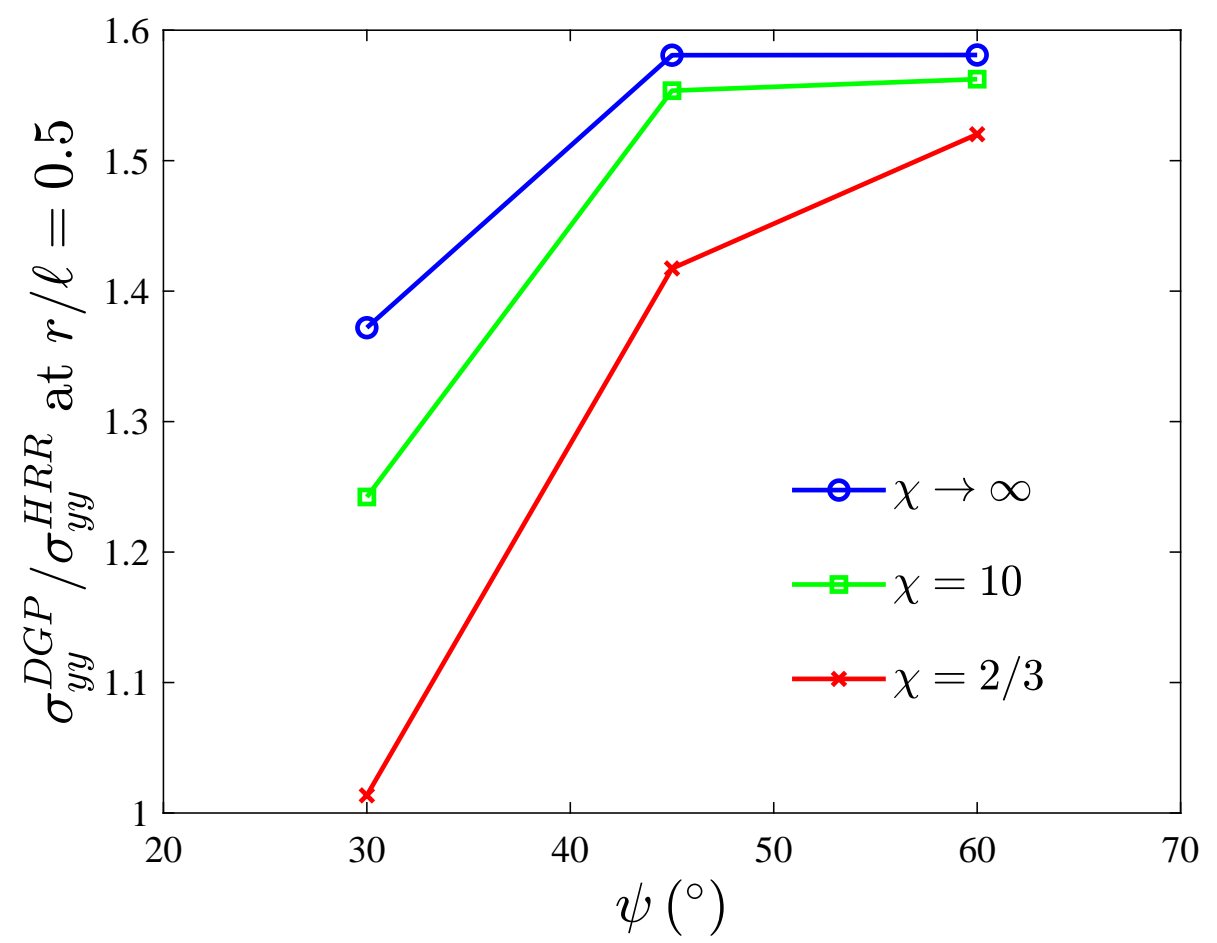

Figure 9: Stress elevation predicted relative to conventional plasticity for selected values of $\chi$ and mode-mix angles. Material properties: $\sigma_{Y} / E=0.003, \nu=0.3, N=0.1$, and $\ell / R_{p}=0.03$.

\subsection{Fracture at bi-material interfaces}

We proceed to investigate cleavage in the presence of significant plastic flow at bi-material interfaces, a paradigmatic conundrum in metallic fracture. Specifically, we reproduce the classic experiments by Elssner et al. [48] and Korn et al. [49] on niobium-sapphire interfaces. Our goal is to properly characterise the interface between elastic and elastic-plastic solids by 
incorporating, for the first time in fracture, the role of suitable higher order boundary conditions to model dislocation blockage.

In a remarkable series of experiments, Elssner et al. [48] and Korn et al. [49] measured both the macroscopic work of fracture and the atomic work of separation of an interface between sapphire and single crystal niobium. The macroscopic toughness turned out to be 1000 times higher than the atomic work of separation, with the difference being attributed to the significant dislocation activity observed in the $\mathrm{Nb}$ single crystal. However, fracture occurred by cleavage, with the crack tip remaining atomistically sharp. Since the stress level required to trigger atomic decohesion of a lattice or a strong interface is more than twice the maximum stress around the crack tip predicted by conventional $J_{2}$ plasticity, the findings by Elssner et al. [48] and Korn et al. [49] constitute a paradox in the context of conventional plasticity [64 66]. We hypothesize that strain gradient effects, combined with dislocation blockage, will be sufficient to raise crack tip stresses beyond the theoretical strength of the metal, $\approx 10 \sigma_{Y}$, over a sufficiently large distance to trigger fracture. The geometry, configuration and dimensions (in $\mathrm{mm}$ ) of the four-point bending experiment Korn et al. [49] are shown in Fig. 10, A sapphire layer is sandwiched between a single crystal and a polycrystalline $\mathrm{Nb}$ layers, which are in turn sandwiched by two alumina layers. An initial crack of length $a=0.4 \mathrm{~mm}$ is placed at the interface between the single crystal $\mathrm{Nb}$ and sapphire. A load of $F=85 \mathrm{~N}$ is applied. 


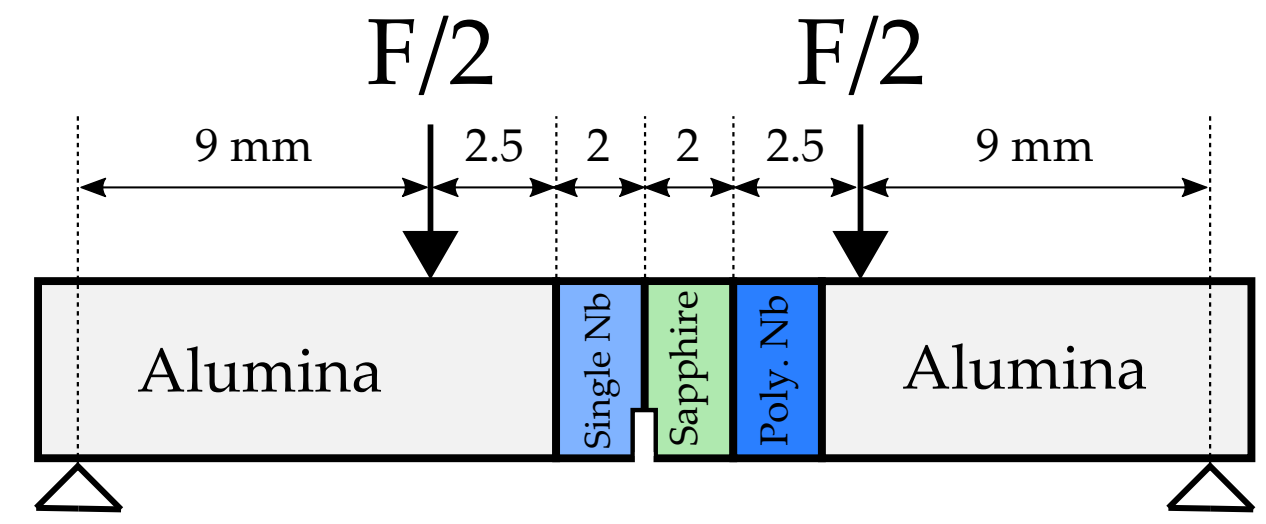

Figure 10: Geometry and dimensions of the four-point bending experiments by Korn et al. 49]. All dimensions are in $\mathrm{mm}$. The polycrystalline Nb layer has identical dimensions to the single crystal $\mathrm{Nb}$ and the sapphire layers. The specimen thickness (in the out-of-plane direction) is $2 \mathrm{~mm}$.

Regarding the material properties, the alumina and the sapphire are linear elastic; alumina has a Young's modulus of $E=390 \mathrm{GPa}$ and a Poisson's ratio of $\nu=0.27$, while sapphire has a Young's modulus of $E=425 \mathrm{GPa}$ and a Poisson's ratio of $\nu=0.16$ [49]. The polycrystalline niobium has a Young's modulus of $E=105 \mathrm{GPa}$, a Poisson's ratio of $\nu=0.39$, a yield stress of $\sigma_{Y}=105 \mathrm{MPa}$ and a strain hardening exponent of $N=0.24$ [49, 65]. Furthermore, the single crystal niobium layer is characterised by a Young's modulus of $E=145 \mathrm{GPa}$, a Poisson's ratio of $\nu=0.36$, and a yield stress of $\sigma_{Y}=145 \mathrm{MPa}$; as in Ref. [65], the strain hardening exponent is chosen to be $N=0.05$ to represent easy glide in single crystal deformation. The magnitude of the material length scales remains to be defined. To the best of the authors' knowledge, no micro-scale experiments have been conducted on single crystal or polycrystalline $\mathrm{Nb}$. A literature review of the experimental works conducted together with a gradient plasticity analysis to obtain the 
material length scales is provided in Table 1.

Table 1: Compilation of experimentally reported length scales with their associated gradient plasticity formulation.

\begin{tabular}{|c|c|c|c|}
\hline Work & Material & Experiment & Length scale - Gradient model \\
\hline Fleck et al. [2] & $\mathrm{Cu}$ & Torsion & $3.7 \mu m$ - Fleck \& Hutchinson (1993) [9] \\
\hline \multirow[t]{2}{*}{ Nix and Gao [3] } & $\mathrm{Cu}$ (cold worked) & Indentation & $5.84 \mu m-$ Nix \& Gao (1998) [3] \\
\hline & Single crystal $\mathrm{Cu}$ & Indentation & $12 \mu m-\operatorname{Nix} \&$ Gao (1998) [3] \\
\hline Stölken and Evans [4] & $\mathrm{Ni}$ & Bending & $5.2 \mu m$ - Fleck \& Hutchinson (1993) [9] \\
\hline Shrotriya et al. [67] & $\mathrm{Ni}$ & Bending & $5.6 \mu m$ - Fleck \& Hutchinson (1993) [9] \\
\hline Haque and Saif [68] & $\mathrm{Al}$ & Bending & $4.5 \mu m$ - Gao et al. (1999) [69] \\
\hline Ro et al. [70] & $\mathrm{Al} 2024$ & Indentation & $0.2 \mu m-$ Nix \& Gao (1998) [3] \\
\hline \multirow[t]{2}{*}{ Qian et al. [71] } & Steel S355 & Indentation & $7 \mu m$ - Gao et al. (1999) [69] \\
\hline & Steel S690 & Indentation & $7 \mu m$ - Gao et al. (1999) [69] \\
\hline Guo et al. [72] & $\mathrm{Cu}$ & Torsion & $3 \mu m$ - Fleck \& Hutchinson (1993) [9] \\
\hline \multirow[t]{2}{*}{ Iliev et al. [73] } & In & Indentation & $85.21 \mu m$ - Nix \& Gao (1998) [3] \\
\hline & In & Bending & $93.34 \mu m$ - Fleck \& Hutchinson (1993) [9] \\
\hline $\mathrm{Mu}$ et al. [74] & $\mathrm{Cu}$ & Micro-pillar shear & $0.647 \mu m$ - Fleck \& Hutchinson (1997) [24] \\
\hline
\end{tabular}

As in Section 5.1, we consider a reference length scale $L_{E}=L_{D}=\ell$ for simplicity. We follow Qu et al. [65] and consider the magnitude of $\ell$ for polycrystalline $\mathrm{Nb}$ to be equal to $5.29 \mu \mathrm{m}$. This value is close to the average magnitude of the length scale measured for $\mathrm{Cu}, \mathrm{Ni}$ and $\mathrm{Al}$ using torsion, indentation and bending. Regarding single crystal $\mathrm{Nb}$, the work by Nix and Gao [3] on $\mathrm{Cu}$ shows that experiments on single crystal samples are best captured with a length scale that duplicates the magnitude of the length scale 
employed to fit the tests on polycrystalline samples (see Table 11). Accordingly, we chose to assume a length scale for single crystal $\mathrm{Nb}$ of $\ell=10.58 \mu \mathrm{m}$. For both single crystal and polycrystalline $\mathrm{Nb}$ the parameter governing dissipation due to the plastic spin is assumed to be equal to $\chi=2 / 3$. The finite element model is constructed using user defined elements, for the elasticplastic materials, and ABAQUS in-built elements for the elastic materials. A total of 20,336 quadrilateral quadratic elements with full integration are used, with the mesh being very refined close to the crack tip - see Fig. 11. The characteristic length of the elements close to the crack tip is of $10 \mathrm{~nm}$. Plane strain conditions are assumed.
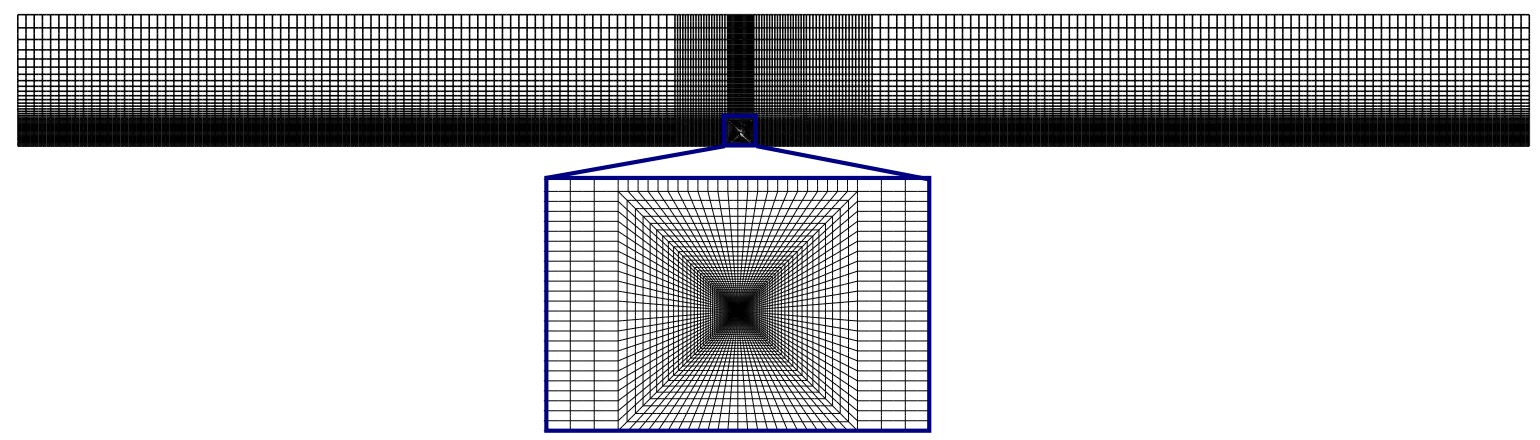

Figure 11: Schematic and detailed views of the finite element mesh employed to model the four-point bending experiments by Korn et al. [49].

The higher order boundary conditions require special consideration. We assume that the interaction between the dislocations and the various material interfaces in the bending specimen is such that dislocations are not allowed to exit the plastic layers. These micro-hard conditions, emulating dislocation blockage, are likely to be a good approximation to the dislocation behaviour at the niobium-sapphire and niobium-alumina interfaces. The degrees of freedom corresponding to the plastic strain tensor and the plastic 
spin are therefore constrained $\varepsilon_{x}^{p}=\varepsilon_{y}^{p}=\varepsilon_{x y}^{p}=\vartheta_{x y}^{p}=0$. Apart from that, the conventional boundary conditions are straightforward, as provided in Fig. 10,

The results obtained from the finite element model are shown in Fig. 12 , in terms of tensile stress versus distance along the interface, ahead of the crack tip. The stress distribution is normalised by the yield stress of single crystal niobium and results are shown for both conventional and distortion gradient plasticity. While the maximum stress predicted by conventional plasticity is below $4 \sigma_{Y}$, insufficient to trigger brittle fracture, the stress level predicted with distortion gradient plasticity exceeds the theoretical lattice strength $\left(10 \sigma_{Y}\right)$ over hundreds of nanometres. Consequently, the combination of local crack tip strengthening and dislocation blockage provides a rational basis for atomic decohesion at bi-material interfaces in the presence of plasticity. 


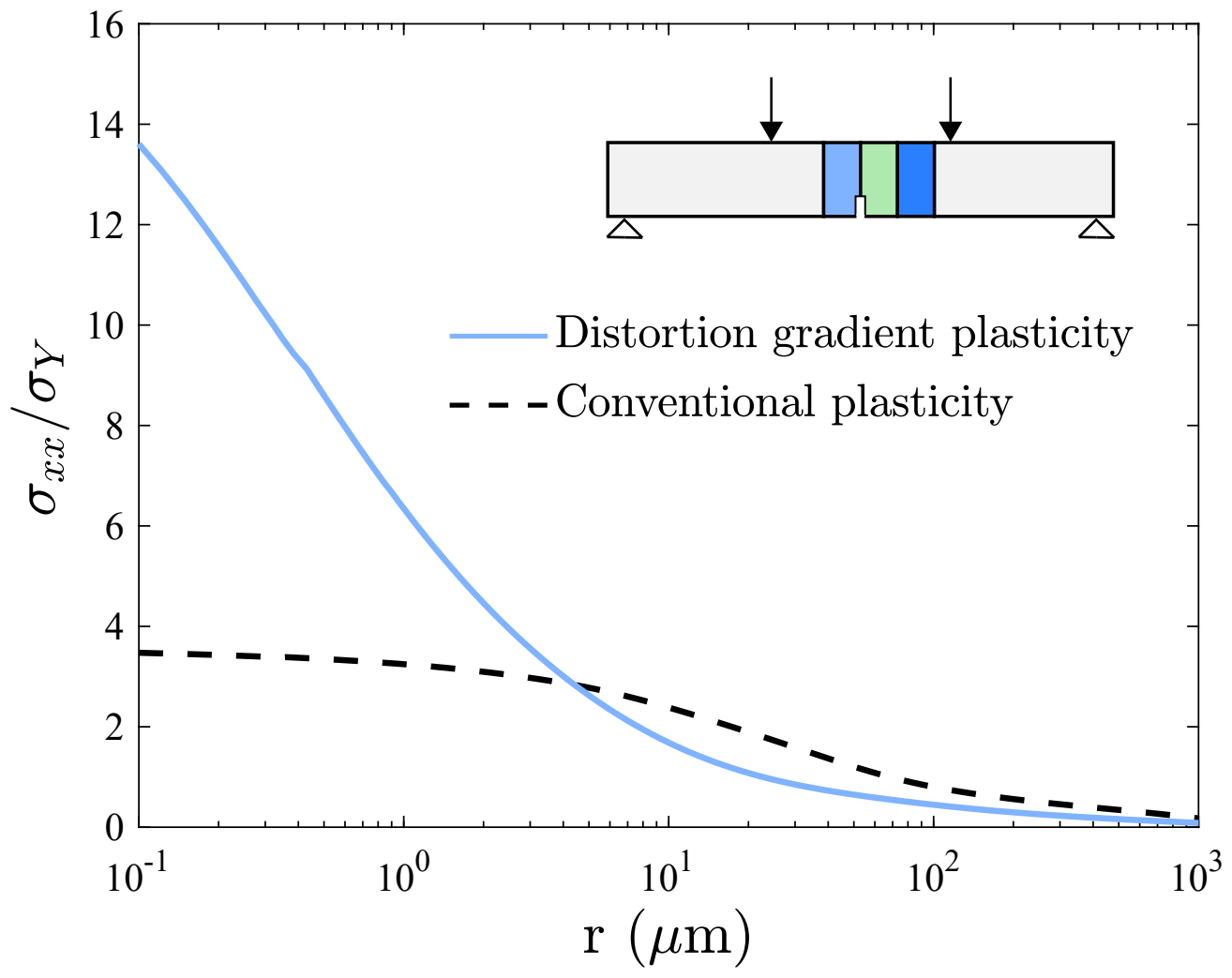

Figure 12: Tensile stress distribution along the interface between single crystal $\mathrm{Nb}$ and sapphire ahead of the crack. The results are shown for distortion gradient plasticity (solid line) and for conventional plasticity (dashed line). The stress distribution is normalised by the yield stress of single crystal $\mathrm{Nb}$.

Finally, contours are obtained for the relevant component of Nye's tensor, as shown in Fig. 13. The magnitude of $\alpha_{x z}$ increases in the vicinity of the crack tip, reaching a maximum value that does not exceed $0.01 \mu \mathrm{m}^{-1}$. This is in agreement with experimental observations of lattice distortions beneath nano-indents - see Ref. [63]. 


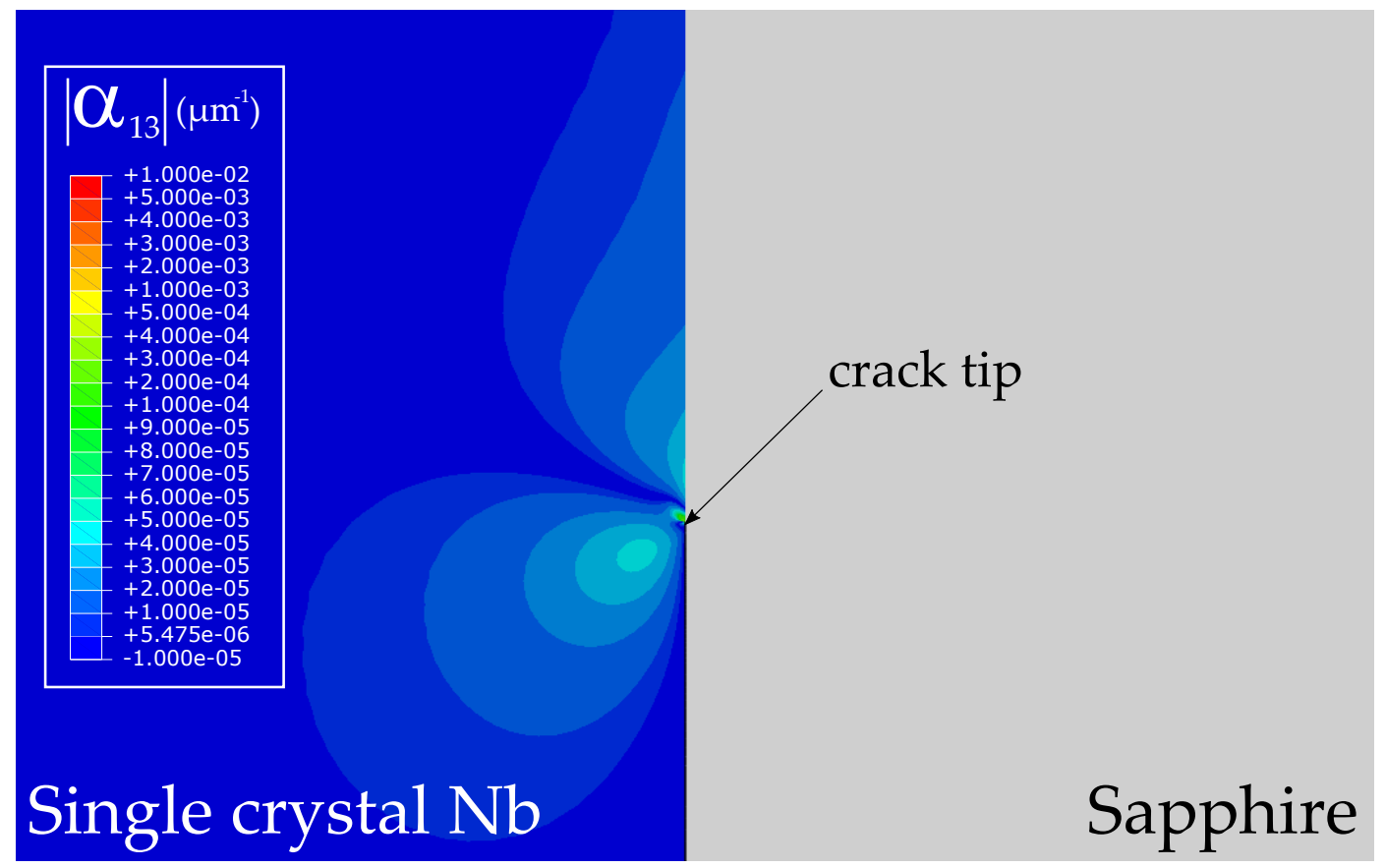

Figure 13: Contours of the relevant component of Nye's tensor, $\alpha_{x z}$, in the $\mathrm{Nb}$ layer in the vicinity of the crack tip.

\section{Conclusions}

We investigate, numerically and analytically, the crack tip behaviour of metals by using distortion gradient plasticity. The influence of two notable constitutive features on fracture mechanics predictions is investigated for the first time: (i) the use of Nye's tensor as primal higher order kinematic variable, and (ii) the role of the plastic spin. A generalised $J$-integral is defined, which is then used to determine the crack tip asymptotic singularity order. On the numerical side, a finite element framework is presented, which builds upon a novel viscoplastic function that enables efficient modelling of both rate-dependent and rate-independent behaviour. The analysis of 
crack tip fields under mode I and mixed-mode fracture assuming small scale yielding conditions reveals the following main findings:

- An elastic region exists close to the crack tip, where the plastic strains are negligible and the Cauchy stress follows the $r^{-1 / 2}$ singularity of linear elasticity. This elastic core, reminiscent of a dislocation-free zone, is present for both gradient-based and curl-based higher order constitutive choices. However, the nature of the singularity will change for a less-than-quadratic defect energy.

- The stress elevation due to strain gradient hardening predicted in the annular plastic zone embedding the elastic core is very sensitive to the constitutive choice of the defect energy. The use of Nye's tensor leads to a substantially weaker stress elevation, as compared with the plastic strain gradient tensor, for the same value of the material length scale. The values of Nye's tensor predicted in the vicinity of the crack are consistent with experimental observations.

- A small influence of $\chi$, the parameter governing the dissipation due to the plastic spin, is observed. Increasing $\chi$ raises crack tip stresses, with the upper bound being given by the irrotational plastic flow scenario.

We emphasise that, for the case where gradients effects are due to Nye's tensor only $\left(L_{D}=0\right)$, our analysis is constrained by the assumption of a continuous plastic distortion field. The framework should be extended to admit discontinuity in some components of the plastic distortion, as in [34], and this will be the goal of future work. 
Finally, the framework is employed to shed light into the paradox of brittle fracture in the presence of plasticity in bi-material interfaces. By modelling the paradigmatic experiments by Elssner et al. [48] and Korn et al. [49] on niobium-sapphire interfaces, we find that:

- The combination of micro-hard higher order boundary conditions, emulating dislocation blockage, and gradient plasticity effects lead to interface crack tip stresses that are larger than the theoretical lattice strength over a distance of hundreds of $\mathrm{nm}$; rationalising quasi-cleavage in bi-material interfaces.

\section{Acknowledgments}

Helpful discussions with Konstantinos Poulios (Technical University of Denmark) and Ivan Moyano (University of Cambridge) are gratefully acknowledged. E. Martínez-Pañeda acknowledges financial support from the People Programme (Marie Curie Actions) of the European Union's Seventh Framework Programme (FP7/2007-2013) under REA grant agreement $n^{\circ}$ 609405 (COFUNDPostdocDTU).

\section{Appendix A. Additional details of numerical implementation}

Assume 2D plane strain conditions, as in the numerical examples addressed in the paper. Accordingly, for an element with $k$ nodes, the nodal variables read,

$$
\begin{aligned}
& \hat{\boldsymbol{u}}=\left[\begin{array}{lllll}
\hat{u}_{x}^{(1)} & \hat{u}_{y}^{(1)} & \cdots & \hat{u}_{x}^{(k)} & \hat{u}_{y}^{(k)}
\end{array}\right]^{T} \\
& \hat{\boldsymbol{\varepsilon}}^{p}=\left[\begin{array}{lllllll}
\hat{\varepsilon}_{x}^{p(1)} & \hat{\varepsilon}_{y}^{p(1)} & \hat{\gamma}_{x y}^{p(1)} & \cdots & \hat{\varepsilon}_{x}^{p(k)} & \hat{\varepsilon}_{y}^{p(k)} & \hat{\gamma}_{x y}^{p(k)}
\end{array}\right]^{T}
\end{aligned}
$$




$$
\hat{\vartheta}_{x y}^{p}=\left[\begin{array}{lll}
\hat{\vartheta}_{x y}^{p(1)} & \cdots & \hat{\vartheta}_{x y}^{p(k)}
\end{array}\right]^{T}
$$

The shape functions matrices for a given node $n$ are then given by,

$$
\boldsymbol{N}_{n}^{u}=\left[\begin{array}{cc}
N_{n} & 0 \\
0 & N_{n}
\end{array}\right] ; \quad \boldsymbol{N}_{n}^{\varepsilon^{p}}=\left[\begin{array}{ccc}
N_{n} & 0 & 0 \\
0 & N_{n} & 0 \\
-N_{n} & -N_{n} & 0 \\
0 & 0 & N_{n}
\end{array}\right]
$$

with $\boldsymbol{N}_{n}^{\vartheta^{p}}$ being, in plane strain conditions, the scalar $N_{n}$ for node $n$. While the interpolation matrices for gradient and curl-based quantities are given by,

$$
\boldsymbol{B}_{n}^{u}=\left[\begin{array}{cc}
\frac{\partial N_{n}}{\partial x} & 0 \\
0 & \frac{\partial N_{n}}{\partial y} \\
0 & 0 \\
\frac{\partial N_{n}}{\partial y} & \frac{\partial N_{n}}{\partial x}
\end{array}\right] ; \quad \boldsymbol{B}_{n}^{\varepsilon^{p}}=\left[\begin{array}{ccc}
\frac{\partial N_{n}}{\partial x} & 0 & 0 \\
\frac{\partial N_{n}}{\partial y} & 0 & 0 \\
0 & \frac{\partial N_{n}}{\partial x} & 0 \\
0 & \frac{\partial N_{n}}{\partial y} & 0 \\
-\frac{\partial N_{n}}{\partial x} & -\frac{\partial N_{n}}{\partial x} & 0 \\
-\frac{\partial N_{n}}{\partial y} & -\frac{\partial N_{n}}{\partial y} & 0 \\
0 & 0 & \frac{\partial N_{n}}{\partial x} \\
0 & 0 & \frac{\partial N_{n}}{\partial y}
\end{array}\right]
$$

and,

$$
\boldsymbol{M}_{n}^{\varepsilon^{p}}=\left[\begin{array}{ccc}
-\frac{\partial N_{n}}{\partial y} & 0 & \frac{1}{2} \frac{\partial N_{n}}{\partial x} \\
0 & \frac{\partial N_{n}}{\partial x} & -\frac{1}{2} \frac{\partial N_{n}}{\partial y} \\
-\frac{\partial N_{n}}{\partial y} & -\frac{\partial N_{n}}{\partial y} & 0 \\
\frac{\partial N_{n}}{\partial x} & \frac{\partial N_{n}}{\partial x} & 0
\end{array}\right] ; \quad \boldsymbol{M}_{n}^{\vartheta^{p}}=\left[\begin{array}{c}
\frac{\partial N_{n}}{\partial x} \\
\frac{\partial N_{n}}{\partial y} \\
0 \\
0
\end{array}\right]
$$


On the other side, the stiffness matrix components are given by,

$$
\begin{aligned}
& \boldsymbol{K}_{n m}^{u, u}=\frac{\partial \boldsymbol{R}_{n}^{u}}{\partial \boldsymbol{u}_{m}}=\int_{\Omega}\left(\boldsymbol{B}_{n}^{\boldsymbol{u}}\right)^{T} \boldsymbol{C} \boldsymbol{B}_{m}^{u} \mathrm{~d} V \\
& \boldsymbol{K}_{n m}^{u, \varepsilon^{p}}=\frac{\partial \boldsymbol{R}_{n}^{u}}{\partial \boldsymbol{\varepsilon}_{m}^{p}}=-\int_{\Omega}\left(\boldsymbol{B}_{n}^{u}\right)^{T} \boldsymbol{C} \boldsymbol{N}_{m}^{\varepsilon^{p}} \mathrm{~d} V \\
& \boldsymbol{K}_{n m}^{\varepsilon^{p}, u}=\frac{\partial \boldsymbol{R}_{n}^{\varepsilon^{p}}}{\partial \boldsymbol{u}_{m}}=-\int_{\Omega}\left(\boldsymbol{N}_{n}^{\varepsilon^{p}}\right)^{T} \boldsymbol{C} \boldsymbol{B}_{m}^{u} \mathrm{~d} V \\
& \boldsymbol{K}_{n m}^{\varepsilon^{p}, \varepsilon^{p}}=\frac{\partial \boldsymbol{R}_{n}^{\varepsilon^{p}}}{\partial \boldsymbol{\varepsilon}_{m}^{p}}=\int_{\Omega}\left\{\left(\boldsymbol{N}_{n}^{\varepsilon^{p}}\right)^{T}\left[\left(\frac{\partial \boldsymbol{q}}{\partial \varepsilon_{m}^{p}}+\boldsymbol{C}\right) \boldsymbol{N}_{m}^{\varepsilon^{p}}+\frac{\partial \boldsymbol{q}}{\partial \nabla \varepsilon_{m}^{p}} \boldsymbol{B}_{m}^{\varepsilon^{p}}\right]\right. \\
& \left.+\left(\boldsymbol{B}_{n}^{\varepsilon^{p}}\right)^{T}\left(\frac{\partial \boldsymbol{\tau}}{\partial \varepsilon_{m}^{p}} \boldsymbol{N}_{m}^{\varepsilon^{p}}+\frac{\partial \boldsymbol{\tau}}{\partial \nabla \varepsilon_{m}^{p}} \boldsymbol{B}_{m}^{\varepsilon^{p}}\right)+\left(\boldsymbol{M}_{n}^{\varepsilon^{p}}\right)^{T} \frac{\partial \boldsymbol{\zeta}}{\partial \alpha_{m}} \boldsymbol{M}_{m}^{\varepsilon^{p}}\right\} \mathrm{d} V \\
& \boldsymbol{K}_{n m}^{\varepsilon^{p}, \vartheta^{p}}=\frac{\partial \boldsymbol{R}_{n}^{\varepsilon^{p}}}{\partial \boldsymbol{\vartheta}_{m}^{p}}=\int_{\Omega}\left[\left(\boldsymbol{N}_{n}^{\varepsilon^{p}}\right)^{T} \frac{\partial \boldsymbol{q}}{\partial \vartheta_{m}^{p}} \boldsymbol{N}_{m}^{\boldsymbol{\vartheta}^{p}}+\left(\boldsymbol{B}_{n}^{\boldsymbol{\varepsilon}^{p}}\right)^{T} \frac{\boldsymbol{\partial} \boldsymbol{\tau}}{\partial \vartheta_{m}^{p}} \boldsymbol{N}_{m}^{\boldsymbol{\vartheta}^{p}}+\left(\boldsymbol{M}_{n}^{\boldsymbol{\varepsilon}^{p}}\right)^{T} \frac{\partial \zeta}{\partial \alpha_{m}} \boldsymbol{M}_{m}^{\boldsymbol{\vartheta}^{p}}\right] \mathrm{d} V \\
& \boldsymbol{K}_{n m}^{\vartheta^{p}, \varepsilon^{p}}=\frac{\partial \boldsymbol{R}_{n}^{\vartheta^{p}}}{\partial \boldsymbol{\varepsilon}_{m}^{p}}=\int_{\Omega}\left[\left(\boldsymbol{N}_{n}^{\boldsymbol{\vartheta}^{p}}\right)^{T}\left(\frac{\partial \boldsymbol{\omega}}{\partial \varepsilon_{m}^{p}} \boldsymbol{N}_{m}^{\varepsilon^{p}}+\frac{\partial \boldsymbol{\omega}}{\partial \nabla \varepsilon_{m}^{p}} \boldsymbol{B}_{m}^{\varepsilon^{p}}\right)+\left(\boldsymbol{M}_{n}^{\boldsymbol{\vartheta}^{p}}\right)^{T} \frac{\partial \zeta}{\partial \alpha_{m}} \boldsymbol{M}_{m}^{\varepsilon^{p}}\right] \mathrm{d} V \\
& \boldsymbol{K}_{n m}^{\vartheta^{p}, \vartheta^{p}}=\frac{\partial \boldsymbol{R}_{n}^{\boldsymbol{\vartheta}^{p}}}{\partial \boldsymbol{\vartheta}_{m}^{p}}=\int_{\Omega}\left[\left(\boldsymbol{N}_{n}^{\boldsymbol{\vartheta}^{p}}\right)^{T} \frac{\partial \boldsymbol{\omega}}{\partial \vartheta_{m}^{p}} \boldsymbol{N}_{m}^{\boldsymbol{\vartheta}^{p}}+\left(\boldsymbol{M}_{n}^{\boldsymbol{\vartheta}^{p}}\right)^{T} \frac{\partial \zeta}{\partial \alpha_{m}} \boldsymbol{M}_{m}^{\boldsymbol{\vartheta}^{p}}\right] \mathrm{d} V
\end{aligned}
$$

\section{References}

[1] G. Z. Voyiadjis, Y. Song, Strain gradient continuum plasticity theories: Theoretical, numerical and experimental investigations, International Journal of Plasticity 121 (2019) 21-75.

[2] N. A. Fleck, G. M. Muller, M. F. Ashby, J. W. Hutchinson, Strain gradient plasticity: Theory and Experiment, Acta Metallurgica et Materialia 42 (2) (1994) 475-487. 
[3] W. D. Nix, H. J. Gao, Indentation size effects in crystalline materials: A law for strain gradient plasticity, Journal of the Mechanics and Physics of Solids 46 (3) (1998) 411-425.

[4] J. S. Stölken, A. G. Evans, A microbend test method for measuring the plasticity length scale, Acta Materialia 46 (14) (1998) 5109-5115.

[5] M. F. Ashby, The deformation of plastically non-homogeneous materials, Philosophical Magazine 21 (170) (1970) 399-424.

[6] V. L. Berdichevsky, L. Sedov, Dynamic theory of continuously distributed dislocations. Its relation to plasticity theory, Journal of Applied Mathematics and Mechanics 31 (6) (1967) 989-1006.

[7] O. W. Dillon, J. Kratochvil, A strain gradient theory of plasticity, International Journal of Solids and Structures 6 (12) (1970) 1513-1533.

[8] E. C. Aifantis, On the role of gradients in the localization of deformation and fracture, International Journal of Engineering Science 30 (10) (1992) $1279-1299$.

[9] N. A. Fleck, J. W. Hutchinson, A phenomenological theory for strain gradient effects in plasticity, Journal of the Mechanics and Physics of Solids 41 (12) (1993) 1825-1857.

[10] N. A. Fleck, J. W. Hutchinson, A reformulation of strain gradient plasticity, Journal of the Mechanics and Physics of Solids 49 (10) (2001) $2245-2271$. 
[11] P. Gudmundson, A unified treatment of strain gradient plasticity, Journal of the Mechanics and Physics of Solids 52 (6) (2004) 1379-1406.

[12] M. E. Gurtin, L. Anand, A theory of strain-gradient plasticity for isotropic, plastically irrotational materials. Part I: Small deformations, Journal of the Mechanics and Physics of Solids 53 (2005) 1624-1649.

[13] M. I. Idiart, V. S. Deshpande, N. A. Fleck, J. R. Willis, Size effects in the bending of thin foils, International Journal of Engineering Science 47 (11-12) (2009) 1251-1264.

[14] N. A. Fleck, J. R. Willis, A mathematical basis for strain-gradient plasticity theory. Part II: Tensorial plastic multiplier, Journal of the Mechanics and Physics of Solids 57 (7) (2009) 1045-1057.

[15] N. Ohno, D. Okumura, Higher-order stress and grain size effects due to self-energy of geometrically necessary dislocations, Journal of the Mechanics and Physics of Solids 55 (9) (2007) 1879-1898.

[16] A. Garroni, G. Leoni, M. Ponsiglione, Gradient theory for plasticity via homogenization of discrete dislocations, Journal of the European Mathematical Society 12 (5) (2010) 1231-1266.

[17] S. Wulfinghoff, S. Forest, T. Böhlke, Strain gradient plasticity modeling of the cyclic behavior of laminate microstructures, Journal of the Mechanics and Physics of Solids 79 (2015) 1-20.

[18] G. Lancioni, T. Yalçinkaya, A. Cocks, Energy-based non-local plasticity models for deformation patterning, localization and fracture, Proceed- 
ings of the Royal Society A: Mathematical, Physical and Engineering Sciences 471 (2180) (2015).

[19] L. Bardella, A. Panteghini, Modelling the torsion of thin metal wires by distortion gradient plasticity, Journal of the Mechanics and Physics of Solids 78 (2015) 467-492.

[20] A. Panteghini, L. Bardella, C. F. Niordson, A potential for higher-order phenomenological strain gradient plasticity to predict reliable reponse under non-proportional loading, Proceedings of the Royal Society A: Mathematical, Physical and Engineering Sciences 475 (2019) 20190258.

[21] M. E. Gurtin, A gradient theory of small-deformation isotropic plasticity that accounts for the Burgers vector and for dissipation due to plastic spin, Journal of the Mechanics and Physics of Solids 52 (11) (2004) $2545-2568$.

[22] J. M. Burgers, Some considerations on the fields of stresses connected with dislocations in a regular crystal lattice, Koninklijke Nederlandse Akademie van Wetenschappen 42 (1931) (1939) 293-325.

[23] J. F. Nye, Some geometrical relations in dislocated crystals, Acta Metallurgica 1 (2) (1953) 153-162.

[24] N. A. Fleck, J. W. Hutchinson, Strain gradient plasticity, Advances in Applied Mechanics 33 (1997) 295-361.

[25] L. Bardella, A. Giacomini, Influence of material parameters and crystallography on the size effects describable by means of strain gradient 
plasticity, Journal of the Mechanics and Physics of Solids 56 (9) (2008) 2906-2934.

[26] L. Bardella, A comparison between crystal and isotropic strain gradient plasticity theories with accent on the role of the plastic spin, European Journal of Mechanics, A/Solids 28 (3) (2009) 638-646.

[27] L. Bardella, Size effects in phenomenological strain gradient plasticity constitutively involving the plastic spin, International Journal of Engineering Science 48 (5) (2010) 550-568.

[28] L. H. Poh, R. H. Peerlings, The plastic rotation effect in an isotropic gradient plasticity model for applications at the meso scale, International Journal of Solids and Structures 78-79 (2016) 57-69.

[29] E. Bittencourt, A. Needleman, M. E. Gurtin, E. Van der Giessen, A comparison of nonlocal continuum and discrete dislocation plasticity predictions, Journal of the Mechanics and Physics of Solids 51 (2) (2003) $281-310$.

[30] L. H. Poh, Scale transition of a higher order plasticity model - A consistent homogenization theory from meso to macro, Journal of the Mechanics and Physics of Solids 61 (12) (2013) 2692-2710.

[31] L. H. Poh, V. T. Phan, Numerical implementation and validation of a consistently homogenized higher order plasticity model, International Journal for Numerical Methods in Engineering 106 (2016) 454-483.

[32] E. Martínez-Pañeda, C. F. Niordson, L. Bardella, A finite element framework for distortion gradient plasticity with applications to bending of 
thin foils, International Journal of Solids and Structures 96 (2016) 288299.

[33] A. Panteghini, L. Bardella, On the Finite Element implementation of higher-order gradient plasticity, with focus on theories based on plastic distortion incompatibility, Computer Methods in Applied Mechanics and Engineering 310 (2016) 840-865.

[34] A. Panteghini, L. Bardella, On the role of higher-order conditions in distortion gradient plasticity, Journal of the Mechanics and Physics of Solids 118 (2018) 293-321.

[35] Y. Wei, J. W. Hutchinson, Steady-state crack growth and work of fracture for solids characterized by strain gradient plasticity, Journal of the Mechanics and Physics of Solids 45 (8) (1997) 1253-1273.

[36] U. Komaragiri, S. R. Agnew, R. P. Gangloff, M. R. Begley, The role of macroscopic hardening and individual length-scales on crack tip stress elevation from phenomenological strain gradient plasticity, Journal of the Mechanics and Physics of Solids 56 (12) (2008) 3527-3540.

[37] E. Martínez-Pañeda, C. Betegón, Modeling damage and fracture within strain-gradient plasticity, International Journal of Solids and Structures 59 (2015) 208-215.

[38] E. Martínez-Pañeda, C. F. Niordson, On fracture in finite strain gradient plasticity, International Journal of Plasticity 80 (2016) 154-167.

[39] E. Martínez-Pañeda, V. S. Deshpande, C. F. Niordson, N. A. Fleck, The 
role of plastic strain gradients in the crack growth resistance of metals, Journal of the Mechanics and Physics of Solids 126 (2019) 136-150.

[40] E. Martínez-Pañeda, C. F. Niordson, R. P. Gangloff, Strain gradient plasticity-based modeling of hydrogen environment assisted cracking, Acta Materialia 117 (2016) 321-332.

[41] P. K. Kristensen, C. F. Niordson, E. Martínez-Pañeda, A phase field model for elastic-gradient-plastic solids undergoing hydrogen embrittlement, Journal of the Mechanics and Physics of Solids 143 (2020) 104093.

[42] S. Brinckmann, T. Siegmund, Computations of fatigue crack growth with strain gradient plasticity and an irreversible cohesive zone model, Engineering Fracture Mechanics 75 (8) (2008) 2276-2294.

[43] J. D. Pribe, T. Siegmund, V. Tomar, J. J. Kruzic, Plastic strain gradients and transient fatigue crack growth: a computational study, International Journal of Fatigue 120 (2019) 283-293.

[44] X. Qian, S. Zhang, S. Swaddiwudhipong, Calibration of Weibull parameters using the conventional mechanism-based strain gradient plasticity, Engineering Fracture Mechanics 78 (9) (2011) 1928-1944.

[45] E. Martínez-Pañeda, S. Fuentes-Alonso, C. Betegón, Gradient-enhanced statistical analysis of cleavage fracture, European Journal of Mechanics - A/Solids 77 (2019) 103785.

[46] E. Martínez-Pañeda, S. del Busto, C. F. Niordson, C. Betegón, Strain gradient plasticity modeling of hydrogen diffusion to the crack tip, International Journal of Hydrogen Energy 41 (24) (2016) 10265-10274. 
[47] E. Martínez-Pañeda, N. A. Fleck, Mode I crack tip fields: Strain gradient plasticity theory versus J2 flow theory, European Journal of Mechanics - A/Solids 75 (2019) 381-388.

[48] G. Elssner, D. Korn, M. Rühle, The influence of interface impurities on fracture energy of UHV diffusion bonded metal-ceramic bicrystals, Scripta Metallurgica et Materiala 31 (8) (1994) 1037-1042.

[49] D. Korn, G. Elssner, R. M. Cannon, M. Ruhle, Fracture properties of interfacially doped Nb-A12O3 bicrystals: I, fracture characteristics, Acta Materialia 50 (15) (2002) 3881-3901.

[50] J. D. Eshelby, The Continuum Theory of Lattice Defects, Solid State Physics 3 (C) (1956) 79-144.

[51] J. Rice, A path independent integral and the approximate analysis of strain concentration by notches and cracks, Journal of Applied Mechanics 35 (2) (1968) 379-386.

[52] J. W. Hutchinson, Singular behaviour at the end of a tensile crack in a hardening material, Journal of the Mechanics and Physics of Solids 16 (1) (1968) 13-31.

[53] J. R. Rice, G. F. Rosengren, Plane strain deformation near a crack tip in a power-law hardening material, Journal of the Mechanics and Physics of Solids 16 (1) (1968) 1-12.

[54] Z. C. Xia, J. W. Hutchinson, Crack tip fields in strain gradient plasticity, Journal of the Mechanics and Physics of Solids 44 (10) (1996) 1621-1648. 
[55] Y. Huang, L. Zhang, T. F. Guo, K. C. Hwang, Mixed mode near-tip fields for cracks in materials with strain-gradient effects, Journal of the Mechanics and Physics of Solids 45 (3) (1997) 439-465.

[56] J. Y. Chen, Y. Wei, Y. Huang, J. W. Hutchinson, K. C. Hwang, The crack tip fields in strain gradient plasticity: the asymptotic and numerical analyses, Engineering Fracture Mechanics 64 (5) (1999) 625-648.

[57] K. L. Nielsen, C. F. Niordson, A 2D finite element implementation of the Fleck-Willis strain-gradient flow theory, European Journal of Mechanics, A/Solids 41 (2013) 134-142.

[58] K. L. Nielsen, C. F. Niordson, A numerical basis for strain-gradient plasticity theory: Rate-independent and rate-dependent formulations, Journal of the Mechanics and Physics of Solids 63 (1) (2014) 113-127.

[59] A. Needleman, Material rate dependence and mesh sensitivity in localization problems, Computer Methods in Applied Mechanics and Engineering 67 (1) (1988) 69-85.

[60] T. L. Anderson, Fracture Mechanics. Fundamentals and Applications, 3rd Edition, CRC Press, Taylor \& Francis, Boca Raton, 2005.

[61] Z. Suo, C. F. Shih, A. G. Varias, A theory for cleavage cracking in the presence of plastic flow, Acta Metallurgica Et Materialia 41 (5) (1993) $1551-1557$.

[62] K. Danas, V. S. Deshpande, N. A. Fleck, Size effects in the conical indentation of an elasto-plastic solid, Journal of the Mechanics and Physics of Solids 60 (9) (2012) 1605-1625. 
[63] S. Das, F. Hofmann, E. Tarleton, Consistent determination of geometrically necessary dislocation density from simulations and experiments, International Journal of Plasticity 109 (2018) 18-42.

[64] H. Jiang, Y. Huang, Z. Zhuang, K. C. Hwang, Fracture in mechanismbased strain gradient plasticity, Journal of the Mechanics and Physics of Solids 49 (5) (2001) 979-993.

[65] S. Qu, Y. Huang, H. Jiang, C. Liu, P. D. Wu, K. C. Hwang, Fracture analysis in the conventional theory of mechanism-based strain gradient (CMSG) plasticity, International Journal of Fracture 129 (3) (2004) 199220.

[66] Y. Jiang, Y. Wei, J. R. Smith, J. W. Hutchinson, A. G. Evans, First principles based predictions of the toughness of a metal/oxide interface, International Journal of Materials Research 101 (2010) 1-8.

[67] P. Shrotriya, S. M. Allameh, J. Lou, T. Buchheit, W. O. Soboyejo, On the measurement of the plasticity length scale parameter in LIGA nickel foils, Mechanics of Materials 35 (3-6) (2003) 233-243.

[68] M. A. Haque, M. T. A. Saif, Strain gradient effect in nanoscale thin films, Acta Materialia 51 (11) (2003) 3053-3061.

[69] H. Gao, Y. Hang, W. D. Nix, J. W. Hutchinson, Mechanism-based strain gradient plasticity - I. Theory, Journal of the Mechanics and Physics of Solids 47 (6) (1999) 1239-1263.

[70] Y. J. Ro, M. R. Begley, R. P. Gangloff, S. R. Agnew, Effect of aging 
on scale-dependent plasticity in aluminum alloy 2024, Materials Science and Engineering A 435-436 (2006) 333-342.

[71] X. Qian, S. Zhang, S. Swaddiwudhipong, L. Shen, Temperature dependence of material length scale for strain gradient plasticity and its effect on near-tip opening displacement, Fatigue and Fracture of Engineering Materials and Structures 37 (2) (2014) 157-170.

[72] S. Guo, Y. He, J. Lei, Z. Li, D. Liu, Individual strain gradient effect on torsional strength of electropolished microscale copper wires, Scripta Materialia 130 (2017) 124-127.

[73] S. P. Iliev, X. Chen, M. V. Pathan, V. L. Tagarielli, Measurements of the mechanical response of Indium and of its size dependence in bending and indentation, Materials Science and Engineering A 683 (2017) 244-251.

[74] Y. Mu, J. W. Hutchinson, W. J. Meng, Micro-pillar measurements of plasticity in confined $\mathrm{Cu}$ thin films, Extreme Mechanics Letters 1 (2014) 62-69. 